\title{
Optimal path crossing the orientation exclusion zone of a robot with offset wrist
}

\author{
Paul Milenkovic(iD) \\ Department of Electrical and Computer Engineering, University of Wisconsin-Madison, 1415 Engineering Drive, Madison, \\ Wisconsin 53706, USA \\ Email: phmilenk@wisc.edu
}

Received: 16 July 2020; Revised: 31 March 2021; Accepted: 1 April 2021; First published online: 21 June 2021

Keywords: Robotic wrist, Nonspherical, Offset wrist, Turning point, Singularity avoidance, Algorithmic differentiation, Power series expansion

\begin{abstract}
An unexpected path reversal is discovered for a serial robot with an offset-axis wrist over a finite range of proximity to the wrist singularity. This is replicated by a kinematic model. A prior spherical-wrist method transits the singularity with limited joint rate and acceleration under a constant rate of tool traversal. Accurate position is maintained by controlling a small deviation in orientation. Extensions to the method for an offset wrist (1) find the least-maximum deviation, (2) identify and locate where a path reversal occurs, and (3) use this point to control step size in a high-order predictor-correction path following procedure.
\end{abstract}

\section{Introduction}

An offset wrist is revealed to have unexpected behavior when its initial and final joint axes become parallel. This discovery was made when applying a recent method for mitigating excessive joint rates at singularity encounter [1] to a 6 degree-of-freedom (dof) serial robot with this type of wrist. A spherical wrist having all three joints intersecting at a common center may experience a condition where a constant rate of traversal of the robot terminal link - the tool - exceeds a wrist-joint rate limit [2]. This also occurs with an offset wrist. An offset wrist is further revealed to have a condition where traversal of the tool halts instantaneously and then reverses direction. This occurs when bounded robot joint rates require the tool traversal rate to cross through zero.

Such motion is known for a planar slider crank. A piston in a cylinder linked to a crank by a connecting rod is a well-known example. As the crank turns, the piston advances toward top dead center (TDC), reaches zero velocity, and then reverses. For such behavior to be associated with a wrist is unanticipated in prior work on singularity avoidance $[3,4]$, where use with an offset wrist was indicated to be a straightforward application of those methods.

ANSI/RIA R15. 06 (1999) [5] Definition 3.40 states that a robot singularity is a "condition caused by collinear alignment of two or more robot axes resulting in unpredictable robot motion and velocities." The assertion that a robot with an offset wrist does not have a wrist singularity $[6,7]$ is correct inasmuch as the type of offset in question allows the critical pair of joint axes to be parallel but not collinear. For this assertion to be made without qualification, however, further suggests that the path reversal occurring near where those joint axes become parallel is indeed not heretofore known. This location is the notional singularity of an offset wrist. 
The singularities of a serial robot are only a problem for the inverse kinematic transformation. Once singularities are identified and either avoided or otherwise mitigated, the resulting joint-angle time histories may be safely applied to the robot joints. This displaces the tool through the forward kinematic transformation. Such is generally not the case with parallel robots $[8,9,10]$, which are not considered here.

\subsection{Prior methods}

Huang et al. [11] classify methods for mitigating the adverse effects of singularities into (1) damped least-squares regularization of the robot Jacobian $[12,13,14]$, which encompasses tracking eigenvalues to set the regularization coefficient $[15,16]$, (2) null-space control of redundant manipulators $[17,18$, 19, 20], and (3) interpolation of joint angles between inverse-kinematic solutions on each side of the singularity. Chembuly and Vorganti (2017) [21] control a redundant planar robot without the eigenvalue decomposition in the null-space methods. Buss and Kim [22] apply a varying amount of regularization to different eigenvalues to effect greater control near the singularity. Null-space control is also applied to nonredundant manipulators by setting an eigenvalue threshold on close approach to the singularity [23, 24]. With nonredundant manipulators, the regularization and null-space methods produce deviations in both tool position and orientation.

The methods of Huang and Milenkovic [3, 25], Aboaf and Paul [26], Cheng et al. [4], and Milenkovic [1] enforce placement of the tool contact point with the work surface during a constant rate of traversal. A small deviation in the tool orientation is tolerated when limiting joint rates. This capability is useful in seam welding, sealant, and paint application along with other manufacturing operations. Cheng et al. [4] along with Milenkovic [1], however, accomplish this by adjusting the displacement path of the robot to pass directly through the wrist singularity instead of close by. Huang et al. [11] nevertheless classified these as singularity avoidance.

Of the four methods enforcing tool position, Milenkovic [1] is unique in finding for a spherical wrist (1) a path optimized to give the least amount of the peak magnitude of tool orientational deviation occurring along this path (i.e., the least maximum) and (2) the least interval of the traversal over which the tool deviates from its desired orientation when satisfying limits on both joint rate and acceleration. The procedures in the path-planning and execution stages are derived from a high-order path-following algorithm, which was found to have a 10-fold reduction in calculation time [27] over a prior secondorder predictor-corrector [28]. Meeting these objectives for an offset wrist motivates the current study. Furthermore, the singularities of the robot arm are readily avoided by staying within reach of the arm and away from the overhead position [17]. This justifies concentrating on wrist effects.

Differing from other optimizations applied to robot paths [29, 30, 31], the least-maximum optimal path is found by searching for a single robot posture for the path to pass through. The optimal path satisfies multiple stationarity conditions at this location. These conditions are met by a path-following procedure conducting a single-parameter search for a spherical wrist [1]. An offset wrist requires the multiparameter search in Section 3.2. When following the pair of paths outward from the least-maximum point, velocity and acceleration constraints are applied by a numerical integration method [32]. All of these procedures are conducted here by searches along a single displacement parameter of a kinematic chain.

High-order path following employs algorithmic differentiation [33] for directly determining the series coefficients of the inverse kinematic transform. These coefficients have a concise derivation and simple expression leading to rapid calculation at high orders [27, 34]. Conditions on a virtual displacement are applied to this process. These control the redundant degrees of freedom accounting for the allowed tool orientational deviation [1]. More recently, Di Gregorio [35] constrains kinematic redundancy in like manner by distinguishing between virtual and actual path displacements in a series expansion of the inverse kinematics. Those symbolic formulas of derivatives are limited to second order (acceleration) with third order (jerk) stated to "yield big analytic expressions." 
The manner in which velocity and acceleration are limited at singularity encounter with high-order path following also differs from procedures addressing related problems in kinematics. Hassan et al. [36] review the application of neural networks to finding constrained-optimal solutions of redundant inverse kinematic problems. Arguing that such methods are much too computationally intensive for limiting both velocity and acceleration during real-time path updates, Huber and Wollherr [37] apply such constraints to a power-series expansion of the forward kinematic transformation of combined translation and rotation in 3D Euclidian space (SE3). The rotation portion is conducted by the Magnus expansion, which is related to the Baker-Campbell-Hausdorff (B-C-H) expansion [38]. Having its first complete closed-form expression due to Dynkin [39], the B-C-H becomes unwieldy from its rapid growth in number of terms beyond third order. In contrast, velocity and acceleration are limited at singularity encounter using a much simpler expression of the series coefficients for the inverse kinematic transform used in path following. A 10th-order expansion gives a good balance between accuracy and calculation speed.

\subsection{Novel contributions}

In extending the method of Milenkovic [1] to an offset wrist, the current paper (1) discovers heretofore unnoted reversals occurring along paths within a finite range of close approach to the notional singularity of an offset wrist, (2) explains how these reversals can occur in a simplified kinematic model, (3) finds either the inflection on a through path or the turning point marking a path reversal, and (4) continues a displacement path beyond where it is otherwise blocked by a path reversal by applying an optimal deviation in orientation when maintaining precise position. The revealed offset-wrist path reversals are analogous to those associated with maximum extension of the robot arm. There, it is possible to deviate in position from the intended path $[40,41,42]$ to continue a displacement otherwise blocked by the turning point singularity at maximum arm extension [28, 43].

Locating the path inflection, or turning point that can occur for an offset wrist, is important in limiting the length of displacement steps along the actual path followed by the robot. This is the path where tool deviation is kept at zero. These steps do not benefit from mitigation of the effects of the singularity along the avoidance path. This is where a joint rate in the wrist is limited and orientational deviation of the tool is allowed at singularity encounter. Knowing this location not only helps plan an optimal avoidance path, it also obtains rapid series convergence on the actual path prior to the start and after the end of the avoidance path. This is accomplished by keeping the step length on the actual path to a fraction of the distance to either a turning point singularity or an inflection indicating a close approach to a singularity.

Finding the inflection or turning point is a challenge unique to an offset wrist. The singularity locating maneuver [1] places the robot at its wrist singularity with the least orientational deviation of the tool. This location is useful in limiting path-following step sizes on the actual path for a spherical wrist. It is not reliable for an offset wrist, however, where a turning point singularity can occur before this location. The inflection or turning point for an offset wrist therefore needs to be located along the actual path by procedures that do not benefit from allowing orientational deviation to obtain singularity mitigation. The method to locate and identify either type of point is hence a particularly important contribution.

\subsection{Organization of the paper}

Section 2 reviews the extension of a 6-axis robot with virtual joints. The complete 9-joint closed-loop kinematic model of the robot gives its desired tool motion. It allows the tool to deviate in orientation while maintaining precise position. The arrangement of these joints also quantifies a measure of the amount of deviation to be optimized at singularity encounter. Section 3 describes how this kinematic loop is displaced to locate the wrist singularity, plan an optimal avoidance path, and then execute that path in carrying out a preplanned task. New procedures allowing for an offset wrist are indicated.

Results of testing the proposed method in computer simulation are presented in Section 4. These tests reveal the transition between through paths and path reversals along actual displacement paths. 
Those paths are at different degrees of close approach to the notional singularity of an offset wrist. The closeness of approach is varied by tilting the plane of the intended tool motion in small increments. The tests show how the singularity avoidance path bridges isolated segments of the actual path under path-reversal conditions. Furthermore, Section 4.2 offers an approximate kinematic model of how an offset wrist can undergo such a path reversal.

Appendix A extends the guided virtual displacement method [1] to multiple virtual displacements. This capability is required to find the least-maximum tool deviation for an offset wrist as described in Section 3.2. Appendix B offers a refinement to an earlier algorithm finding roots of the displacement path as represented by a power series [1]. This refinement covers more cases of possible root locations. The improved algorithm is used in Section 3.4 to locate the point of inflection or path reversal along the actual path. It is also applied to the path displacement in Section 3.4.2 meeting an acceleration limit.

\section{Role of additional joint axes in singularity avoidance}

Kieffer's [28] numerical inverse-kinematic procedure solves for time histories of the joint rates in a 6axis robot generating a desired tool trajectory. This trajectory is represented by an angular rate applied to virtual joint 7 . The articulation of that joint represents the desired motion of the tool with respect to a base and axis direction fixed in space with respect to ground reference. Such completes a 7-joint kinematic loop, where a predictor-corrector path-following method tracks its displacement.

Milenkovic [1] represents tool displacement in a singularity avoidance procedure for a 6-axis robot with two additional virtual joint axes to complete a 9-joint loop. The physical joints in a robot are numbered 1-6. These are followed by a virtual wrist representing the tolerated orientational deviation of the tool, the joint axes of which intersect at the tool contact point (TCP) with its work surface. The joints in the virtual wrist are numbered 6-8, with physical joint 6 and virtual joint 6 assigned the same joint number because they share a common axis line. Furthermore, the path-following algorithm solves for their combined angle $\theta_{6}$ about this common axis. This angle is separable into $\theta_{6 \mathrm{~W}}$ for the physical joint and $\theta_{6 T}$ for the virtual joint. Finally, virtual joint 9 represents the desired or intended tool motion in relation to ground.

The allowed deviation of the tool, therefore, is that of a spherical joint pivoting about the TCP. The articulation of this joint represented by the spherical virtual wrist does not displace its center point, enforcing positioning of the tool contact point without the iterative pointwise corrections required in other methods $[3,4]$.

Two robots are evaluated, each with a different orientation of the wrist offset. Recent robots include the Yaskawa Motoman-MA1400 and Fanuc M-710iC/50E. The MA1400 offsets the axis line of joint 6 in a perpendicular direction to the axis of joint 5. This affords joint 5 an asymmetric angle range [44], one allowing it to point the tool back along the forearm on one side of its joint-5 deflection arc. This capability avoids workspace obstacles in welding tasks. The $50 \mathrm{E}$ offsets the axis line of joint 6 along the axis line through joint 5. Its manufacturer describes its wrist as offering "super-flexible positioning" and recommends this robot "for grinding and polishing applications." There, the robot grasps the work piece to hold it against a grinding or polishing wheel, where this offset direction allows $380^{\circ}$ of usable deflection on joint 5. An earlier welding robot required this second type of offset wrist for its increased load carrying capability [45]. The MA1400 and 50E robots are thus selected as representative of industrial practice.

Wu et al. depict the MA1400 as carrying a curved tool nearly a half meter long. In the current paper, the tool length on the $50 \mathrm{E}$ is set to a mid-range value between this length and the $0.2 \mathrm{~m}$ distance between wrist center and work piece tested for a spherical wrist [1]. Commercial literature reveals the tool used with the MA1400 to be a welding torch. Both straight and curved torches are commercially available. A curved torch, however, violates the requirement of the proposed method that the tool axis line be collinear with joint 6 . The MA1400 is considered here with a straight torch in accord with the tool 
(a)

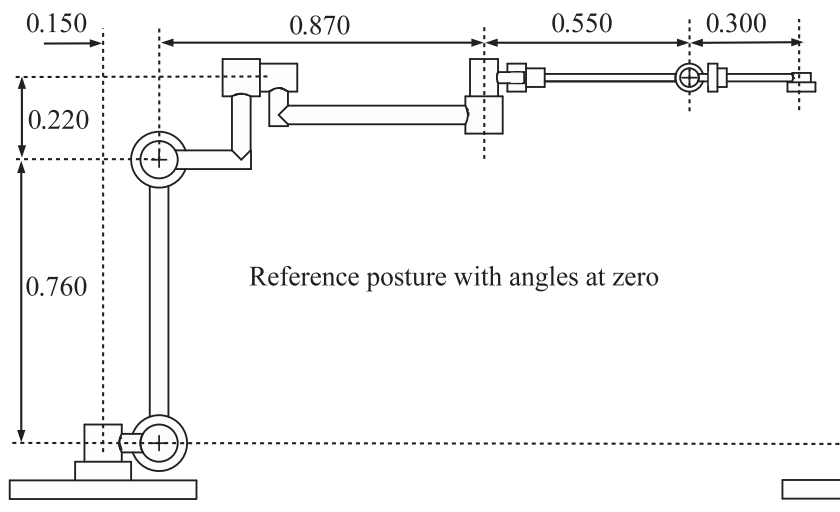

(b)

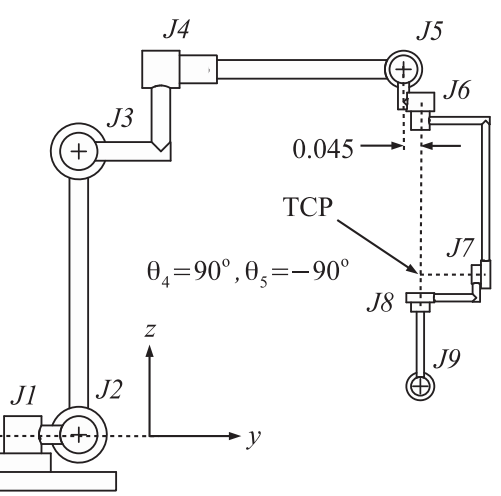

Figure 1. Two postures of the MA1400 robot having an offset wrist. Joint J9 represents the desired task motion of a tool having the specified tool contact point (TCP), with $\mathrm{J} 6, \mathrm{~J} 7$, and $\mathrm{J} 8$ allowing deviation in tool orientation about the TCP.

(a)

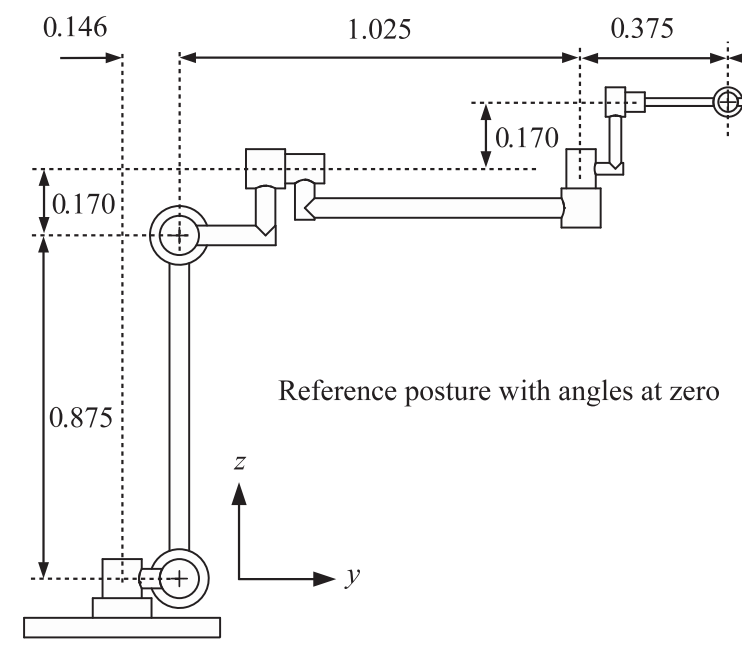

(b)

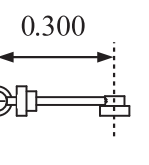

)

)

\section{.}


Table 1. D-H parameters of the MA1400 robot (for the 50E robot, angles $+\theta$ and $\alpha$ are identical, whereas lengths $d$ and $r$ are in parenthesis).

\begin{tabular}{ccccr}
\hline & $+\boldsymbol{\theta}$ & $\boldsymbol{d}(\mathbf{m})$ & $\boldsymbol{r}(\mathbf{m})$ & $\boldsymbol{\alpha}$ \\
\hline$J 1$ & $90^{\circ}$ & & $0.150(0.146)$ & $90^{\circ}$ \\
$J 2$ & $90^{\circ}$ & & $0.760(0.875)$ & \\
$J 3$ & & & $0.220(0.170)$ & $90^{\circ}$ \\
$J 4$ & $-90^{\circ}$ & $0.870(1.025)$ & & $-90^{\circ}$ \\
$J 5$ & & $(0.170)$ & .045 & $90^{\circ}$ \\
$J 6$ & $-90^{\circ}$ & $0.550(0.375)$ & & $90^{\circ}$ \\
$J 7$ & $180^{\circ}$ & & & $90^{\circ}$ \\
$J 8$ & $-90^{\circ}$ & 0.300 & & $-90^{\circ}$ \\
\hline
\end{tabular}

(a)

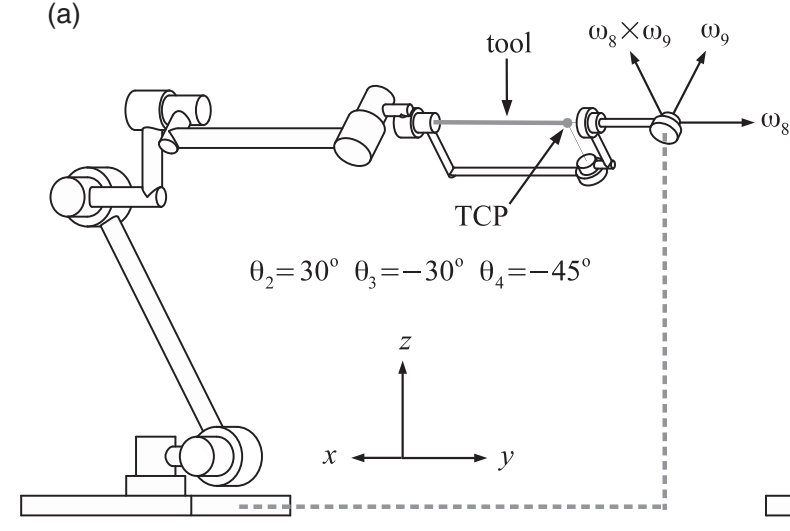

(b)

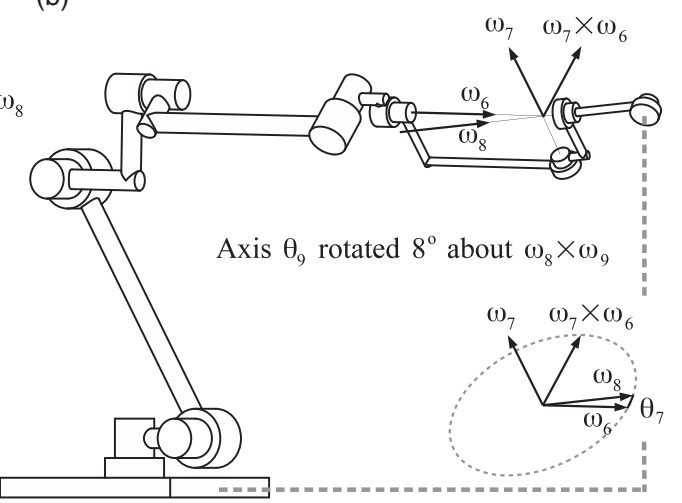

Figure 3. Placing the MA1400 robot in an initial posture in relation to its wrist singularity.

the path inflections and path reversals seen in Figs. 4, 5, 6 and 7 of Section 4 may differ from this position. Cheng et al. [4] describe how for either a spherical or an offset wrist, interpolating a path through $\theta_{5}=0$ avoids a $180^{\circ}$ excursion of $\theta_{4}$. As $\theta_{5}=0$ marks where joints 4 and 6 become parallel, the proposed method in similar fashion redirects the displacement path followed by the wrist to pass through its notional singularity.

Fig. 1(b) shows the MA1400 in the posture illustrated by Wu et al. [7] showing the wrist offset. The dimensions of the MA1400 are from that source. As link dimensions for either robot are not disclosed on manufacturer data sheets, the dimensions of the $50 \mathrm{E}$ were measured from the drawing on its data sheet by using $2 \mathrm{D}$ illustration software. The goal is not absolute precision but rather representative proportions of robots for algorithm testing.

Table 1 gives Denavit-Hartenberg (D-H) parameters for these two robots, with the six joints of each robot augmented with joints 7 and 8 of the virtual wrist followed by joint 9 representing the desired translational and orientation displacement of the tool. Rows of this table reference each numbered joint together with the link that follows it; $+\theta$ gives the value added to the nominal joint angle to direct the local $x$-axis to intersect the next joint-axis line, $d$ is the offset distance along the local $z$-axis of joint rotation, $r$ is the link length along the local $x$-axis to the next joint axis, and $\alpha$ is the angle of rotation about this $x$-axis orienting the $z$-axis of rotation for the next joint. The difference in joint offset directions between robots is manifest in row $J 5$ of the table. The MA1400 wrist offset is along the joint 5 local $x$-axis (parameter $r$ ), whereas the 50E offset is along the joint 5 local $z$-axis of joint rotation (parameter $d$ ).

For the plane containing unit joint axis-direction vectors $\omega_{6}$ and $\omega_{8}$ in Fig. 3(b), rotation by angle $\theta_{7}$ about direction $\omega_{7}$ normal to that plane represents a change in body orientation. Conjugate joint angles $\theta_{6 T}=-\theta_{8}$ in the virtual wrist result in a change in body orientation between the actual and desired 
orientation of the tool, by the same angle of rotation about the same axis but in a rotated coordinate frame. In turn, $\theta_{7}$ is the least angle of rotation about an axis redirecting the axis line of joints 6 to align with joint 8 in any frame, constraining the allowed change in body orientation [1]. Angle $\theta_{7}$ in this way expresses the tool deviation angle with the least change in body orientation meeting this constraint.

The deviation angle $\theta_{7}$ may be minimized by orienting its arc plane depicted in Fig. 3(b). This is done by controlling the combined angle $\theta_{6}$ to rotate axis direction $\boldsymbol{\omega}_{7}$ about $\boldsymbol{\omega}_{6}$. Under the conjugate angle condition $\theta_{6 T}=-\theta_{8}$ and expressing $\theta_{6}=\theta_{6 T}+\theta_{6 W}$, physical joint 6 is actuated by $\theta_{6 W}=\theta_{6}-\theta_{6 T}=$ $\theta_{6}+\theta_{8}$.

\section{Singularity avoidance planning and execution}

In both the path planning and path execution phases, maneuver in close proximity to the singularity of a spherical wrist is conducted by constraining the rate of joint 4 in relation to joint 9 and accepting nonzero tool deviation appearing on joint 7 . Under this condition, the tool is displaced along an adjusted path, achieved by the 6-axis robot and differing from the desired tool displacement along the reference path on account of nonzero $\theta_{7}$. The zero-deviation condition of $\theta_{7}=0$ makes the actual and desired tool displacements the same along the actual path. Segments of the actual path occur before and after making such path adjustments along the singularity avoidance maneuver.

When holding in a constant ratio the rate of joint 4 in the physical wrist and joint 9 representing the tool traversal and with the angle of combined joint 6 controlling the tool deviation angle occurring on joint 7, path-following steps may be conducted with joints 1, 2, 3, 5 in the robot and joints 7 and 8 in the virtual wrist supplying the necessary number of six passive joints to displace a spatial loop. The methods of Milenkovic and Huang [25] as well as Aboaf and Paul [26] effectively fix combined angle $\theta_{6}=0$ without attempting to minimize $\theta_{7}$. Furthermore, joints 5,7 and 8 have axis directions $\omega_{5}=\omega_{7} \times \omega_{6}, \omega_{7}$ and $\omega_{8}$ that are nearly separated by $90^{\circ}$ when tool deviation $\theta_{7}$ is kept well under $90^{\circ}$ (see inset to Fig. 3). This condition supplies a mobile wrist when joint axes $\boldsymbol{\omega}_{4}$ and $\boldsymbol{\omega}_{6}$ are in near alignment, placing the physical wrist with joints 4, 5, and 6 near its singular posture. For the robots under consideration, this condition is observed to be maintained in an offset wrist near its notional singularity.

\subsection{Place the tool on a path encountering the wrist singularity}

In preparation for planning an avoidance path and its subsequent execution, the robot is positioned to make joints 4 and 6 collinear, or parallel in the instance of an offset wrist, and the axis of joint 9 is inclined by the tool deflection angle depicted in Fig. 3(b). Rotation about joint 9 to find a starting point away from this posture, followed by rotation about joint 7 to remove the tool deviation, places the tool on an actual path. Starting from that location, the singularity locating maneuver [1] displaces the tool back to where joints 4 and 6 are aligned with axis directions $\omega_{4}=\omega_{6}$, with $\theta_{6}$ giving a minimum tool deviation $\theta_{7}$.

\subsection{Find the least-maximum tool deviation}

Along a path directed through $\omega_{4}=\omega_{6}$, the least-maximum deviation is the smallest amount of the peak magnitude of the tool deviation angle occurring along all such paths. A necessary condition is for $\theta_{7}$ to be stationary with respect to changes in $\theta_{4}, \theta_{5}$ and $\theta_{6}$. A least-maximum occurs at a saddle point where the minimum $\theta_{7}$ resulting from adjusting $\theta_{6}$ is a maximum over the collection of adjusted paths through all possible pairs $\theta_{4}$ and $\theta_{5}$. Stationarity occurs when joints $1,2,3,7,8$, and 9 are made passive and the instantaneous rate $C_{7}$ of joint 7 is zero when a nonzero rate $C_{4}, C_{5}$, and $C_{6}$ is applied to either of joints 4,5 or 6 . This condition is met at the end of a finite displacement step guiding angles $\theta_{4}, \theta_{5}$, and $\theta_{6}$ by the method in Appendix A. This is a change from a spherical wrist where the least-maximum $\theta_{7}$ occurs at $\theta_{5}=0$, reducing the number of stationarity conditions. 
The virtual wrist is singular, however, when tool deviation $\theta_{7}=0$ aligns joints 6 and 8 . This condition is avoided at zero or small $\theta_{7}$ by substituting joint $6 \mathrm{p}$ for 6 in the virtual displacement (see Appendix A) giving stationary $\theta_{7}$. Joint $6 \mathrm{p}$ has axis $\boldsymbol{\omega}_{6 \mathrm{p}}=\boldsymbol{\omega}_{7} \times \boldsymbol{\omega}_{6}$ perpendicular to joints 7 and 6 . The axis line of $6 \mathrm{p}$ passes through the tool center point (TCP, not WCP as stated earlier [1]). The proof [1] that joint $6 \mathrm{p}$ can substitute for joint 6 is correct for the TCP as were subsequent numerical results.

\subsection{Follow paths outward to reduce the tool deviation to zero}

A path is followed outward from $\theta_{4}=\theta_{4 \text { ref }}$ and $\theta_{9}=\theta_{9 \text { ref }}$ at the point of least-maximum deviation on the adjusted path, to intercept the actual path where $\theta_{7}=0$ - see Fig. 7(a) in Section 4.1. This is done both before and after singularity encounter. Differing from the spherical wrist case, successfully reaching segments of the actual path before and after encounter of an offset wrist requires limiting the rate on joint 4 in relation to joint 9 , even if the initiation and termination points on those segments are later adjusted to shorten the path length of the avoidance maneuver.

\subsection{Locate singularities along the actual path}

An offset wrist introduces new restrictions on movement along the actual path before and after singularity encounter. To achieve satisfactory convergence of the high-order series expansion described in Appendix A, path-following steps are limited to $0.3\left(\theta_{9}-\theta_{9 \text { ref }}\right)$. Whereas $\theta_{9 \text { ref }}$ satisfactorily supplies a bound on the region of convergence for a spherical wrist, this is not the case with an offset wrist where a singularity can occur near but not at its notional singularity.

Locating proximity to a singularity along the actual path is problematic when the adjusted path no longer indicates the singularity location needed to limit the step size. The proposed solution follows the actual path upon making joint 9 passive in place of joint 4, using the method of Appendix B to locate where rate $C_{9}$ reaches a minimum, indicating proximity to the type of singularity encountered by a spherical wrist, or crosses through zero, indicating a turning point along a path reversal encountered by an offset wrist. The value $\theta_{9 \text { sing }}$ at this point is recorded. The path steps in this procedure are limited to $0.3\left(\theta_{4}-\theta_{4 \mathrm{ref}}\right)$. The horizontal line marks $\theta_{4 \text { ref }}$ in Figs. 4,6 , and 7 in Section 4 in relation to the asymptotic values of that angle before and after singularity encounter. These results along with low loop closure error at the end of path-following steps confirm that $\theta_{4 \mathrm{ref}}$ supplies a usable bound on the region-of-convergence for conducting steps in $\theta_{4}$.

\subsubsection{Set initial avoidance-maneuver starting point}

The path-following variable reverts to $\theta_{9}$ when making adjustments along the actual path. For an approach to the wrist singularity along a "before" path segment with increasing $\theta_{9}$, \pm 0.3 ( $\left.\min \left(\theta_{9 \text { ref }}, \theta_{9 \text { sing }}\right)-\theta_{9}\right)$ gives a conservative step length for advancing or retreating from the singular region. In seeking the shortest interval for the singularity avoidance maneuver when allowing the highest rate on joint 4 , advance along the actual path is limited to min $\left(\theta_{9 \text { ref }}, \theta_{9 \text { sing }}\right)-0.5^{\circ}$. An angle separation below $0.5^{\circ}$ allows a shorter interval of the adjusted path with nonzero tool deviation in trade for more calculations.

For a maximum tool deviation magnitude below $0.001^{\circ}$, an alternative procedure avoids possible numerical degeneracy. Starting from $\theta_{9 \mathrm{ref}}$ and setting $\theta_{9 \text { sing }}=\theta_{9 \mathrm{ref}}$, the procedure of Section 3.1 reaches $\theta_{9} \leftarrow \theta_{9 \text { sing }}-0.5^{\circ}$ with $\theta_{7}=0$.

\subsubsection{Adjust starting point to meet joint acceleration and rate limits}

The avoidance maneuver is to be conducted at a constant tool traversal rate $C_{9}$ when limits are applied to wrist rate $C_{4}$ and acceleration $\dot{C}_{4}$. Angle $\theta_{9}$ is the path variable for adjusting the starting point along the actual path to meet these limits. In place of separate procedures for coarse and fine corrections [1], the complete adjustment is now made by the procedure of Appendix B. 
To ensure this adjustment is only away from the singularity, especially at a high rate limit on an offset wrist, a third procedure is introduced below to find the acceleration meeting the rate limit without adjusting $\theta_{9}$. Applying $C_{9}=1 \mathrm{rad} / \mathrm{s}$ and when $0<r / s<1$, a prior formula [1] gives

$$
\theta_{4 \text { limit }}=C_{V 4 \max } s-0.5\left(C_{V 4 \max }-C_{V 4}\right) r
$$

where $\theta_{4 \text { limit }}=\theta_{4 \text { ref }}-\theta_{4}, C_{V 4 \text { max }}$ is the $C_{4} / C_{9}$ limit, $C_{V 4}$ is the ratio $C_{4} / C_{9}$ at $\theta_{9}$, path length $s=\theta_{9 \mathrm{ref}}-\theta_{9}$, and $r$ is the path length over which a constant acceleration applied to joint 4 reaches the rate limit. Acceleration is therefore change in rate over path length giving

$$
a_{4 \text { no adjust }}=\frac{C_{V 4 \max }-C_{V 4}}{r}=0.5 \frac{\left(C_{V 4 \max }-C_{V 4}\right)^{2}}{\left(C_{V 4 \max } s-\theta_{4 \text { limit }}\right)}
$$

where angles are expressed in natural units of radians, rates in $\mathrm{rad} / \mathrm{s}$, and acceleration in $\mathrm{rad} / \mathrm{s}^{2}$.

\subsubsection{Adjust before- and after-singularity paths to match peak rate}

The before- and after-singularity halves of the avoidance maneuver meet at $\left(\theta_{9 \text { ref }}, \theta_{4 \text { ref }}\right)$ with largest tool deviation $\theta_{7}$. To achieve continuity of joint rates, the peak rate of joint 4 after applying Section 3.4.2 to the "before" half supplies the rate limit for this procedure applied to the "after" half. If "after" reduces the rate limit further, the adjustment of "before" is repeated. If the before and after rates still disagree, neither rate limit is active. Adjustments are then made to the constant accelerations applied to before and after. This condition is expected to occur for low maximum deviation, but it was not required for any of the cases presented in Section 4.

\subsection{Determine step sizes on the avoidance maneuver}

The avoidance maneuver is conducted at a constant rate of tool traversal applied to joint 9 . Along both halves of the singularity avoidance maneuver meeting at $\left(\theta_{9 \mathrm{ref}}, \theta_{4 \mathrm{ref}}\right)$, constant acceleration is applied to joint 4 until its rate limit is reached, after which the rates of joints 4 and 9 are held in a constant ratio.

In the absence of a known singularity on the adjusted path, fixing constant-rate steps of joint 4 at $20^{\circ}$, however, gave elevated closure error (position $94 \times 10^{-6} \mathrm{~m}$, angle $3324 \times 10^{-6^{\circ}}$ at $-8^{\circ}$ tool deviation for the MA1400). A step limit of $14^{\circ}$ gives the results reported in Tables 2 and 3 in Section 4 without resorting to a variable step size responding to closure error.

Increasing the entry for $d$ in row $J 8$ of Table 1 places the tool-motion pivot point on virtual joint 9 farther away from the robot. This in turn places steps along the avoidance maneuver closer to reaching the turning point of maximum arm extension. Such would require further restrictions on step length during the avoidance maneuver.

The following procedure effectively limited steps conducted at constant acceleration without occurrence of high closure error requiring shortening the step and recalculating. The sine and cosine functions of displacement generated by joint rotation have an infinite radius of series convergence, but consider $1 \mathrm{rad}\left(57.3^{\circ}\right)$ to be its effective value where convergence becomes impractically slow. A maximum allowed step $s$ is calculated for either direction that changes $\theta_{4}$ by $\pm 1 \mathrm{rad}$. This is expressed as the solution to $\dot{C}_{4} s^{2} / 2+C_{4} s \pm 1=0$ at acceleration $a=\dot{C}_{4}$ and rate $v=C_{4}$. For $\alpha=\left|C_{4} / \dot{C}_{4}\right|, g=\left|2 / \dot{C}_{4}\right|$, the smallest positive-valued step length meeting these conditions is

$$
\begin{aligned}
& s_{1}=\left|-\alpha+\sqrt{\alpha^{2}+g}\right|, \\
& \text { if } \alpha^{2}<g, \quad s=s_{1} \\
& \text { otherwise, } \quad s=\min \left(s_{1},\left|-\alpha+\sqrt{\alpha^{2}-g}\right|\right)
\end{aligned}
$$

The step length limit is subsequently set to $0.3 \mathrm{~s}$. This limit is found to give low closure error with a small number of steps over a wide range of acceleration and rate limits. 
Table 2. Singularity avoidance paths of MA1400 robot: wrist offset $0.045 \mathrm{~m}$, tool length $0.55 \mathrm{~m}$.

\begin{tabular}{|c|c|c|c|c|c|c|c|}
\hline \multicolumn{2}{|c|}{ Tool angle $\left({ }^{\circ}\right)$} & \multirow[b]{2}{*}{ Actual path } & \multicolumn{3}{|c|}{ Avoidance $\theta_{\mathrm{A} 9}\left({ }^{\circ}\right)$} & \multicolumn{2}{|c|}{ Loop closure error } \\
\hline Deflection & Deviation & & Limit $C_{4} / C_{9}$ & Start & End & $\begin{array}{l}\text { Position } \\
\left(10^{-6} \mathrm{~m}\right)\end{array}$ & $\begin{array}{c}\text { Angle } \\
\left(10^{-6 \circ}\right)\end{array}$ \\
\hline-8 & -9.476 & Through & 6 & -8.3 & 7.0 & 1.071 & 39.637 \\
\hline-4 & -4.634 & Through & 12 & -4.3 & 3.7 & 0.078 & 4.256 \\
\hline-2 & -2.201 & Through & 24 & -2.4 & 2.0 & 0.070 & 4.159 \\
\hline-1 & -0.982 & Through & 48 & -1.5 & 1.3 & 0.034 & 2.111 \\
\hline-0.408 & -0.259 & $1 \mathrm{~s}-$ curve & 34.5 & -1.1 & 0.9 & 0.010 & 0.605 \\
\hline-0.289 & -0.114 & $2 \mathrm{~s}-$ curves & 20.2 & -0.9 & 0.9 & 0.005 & 0.279 \\
\hline-0.197 & -0.001 & $2 \mathrm{~s}-$ curves & 0.5 & -0.5 & 0.5 & 0.000 & 0.006 \\
\hline-0.196 & 0.000 & Bifurcated & -0.1 & -0.5 & 0.5 & 0.000 & 0.003 \\
\hline-0.195 & 0.001 & Reversal & -0.7 & -0.5 & 0.5 & 0.000 & 0.004 \\
\hline 0.49 & 0.838 & Reversal & -48 & -1.0 & 1.1 & 0.039 & 2.479 \\
\hline 0.5 & 0.850 & $2 \mathrm{~s}-$ curves & -48 & -1.0 & 1.1 & 0.039 & 2.513 \\
\hline 0.594 & 0.965 & $2 \mathrm{~s}-$ curves & -48 & -1.1 & 1.2 & 0.044 & 2.822 \\
\hline 0.698 & 1.092 & $1 \mathrm{~s}$-curve & -48 & -1.1 & 1.2 & 0.049 & 3.160 \\
\hline 2 & 2.686 & Through & -24 & -2.0 & 2.2 & 0.086 & 5.792 \\
\hline 4 & 5.138 & Through & -12 & -3.7 & 4.2 & 0.044 & 2.878 \\
\hline 8 & 10.057 & Through & -6 & -7.3 & 8.2 & 0.032 & 2.808 \\
\hline
\end{tabular}

Table 3. Singularity avoidance paths of 50 E robot: wrist offset $0.170 \mathrm{~m}$, tool length $0.375 \mathrm{~m}$.

\begin{tabular}{|c|c|c|c|c|c|c|c|}
\hline \multicolumn{2}{|c|}{ Tool angle $\left(^{\circ}\right)$} & \multirow[b]{2}{*}{ Actual path } & \multirow[b]{2}{*}{ Limit $C_{4} / C_{9}$} & \multicolumn{2}{|c|}{ Avoidance $\theta_{\mathrm{A} 9}\left({ }^{\circ}\right)$} & \multicolumn{2}{|c|}{ Loop closure error } \\
\hline Deflection & Deviation & & & Start & End & $\begin{array}{l}\text { Position } \\
\left(10^{-6} \mathrm{~m}\right)\end{array}$ & $\begin{array}{c}\text { Angle } \\
\left(10^{-6 \circ}\right)\end{array}$ \\
\hline-8 & -9.821 & Through & 3 & -11.0 & 9.9 & 1.563 & 107.137 \\
\hline-4 & -4.977 & Through & 3 & -7.9 & 7.3 & 0.593 & 21.700 \\
\hline-2 & -2.543 & Through & 6 & -3.8 & 3.5 & 0.032 & 1.814 \\
\hline 0.082 & -0.001 & Through & 0.5 & -0.5 & 0.5 & 0.000 & 0.003 \\
\hline 0.083 & 0.000 & Bifurcated & -0.1 & -0.5 & 0.5 & 0.000 & 0.000 \\
\hline 0.084 & 0.001 & Reversal & -0.6 & -0.6 & 0.6 & 0.000 & 0.003 \\
\hline 2 & 2.347 & Reversal & -18.9 & -5.6 & 4.4 & 0.037 & 1.615 \\
\hline 4 & 4.801 & Reversal & -23.1 & -7.3 & 4.9 & 0.050 & 3.392 \\
\hline 6.207 & 7.516 & Reversal & -27.7 & -8.2 & 4.7 & 0.031 & 3.500 \\
\hline 8 & 9.726 & Reversal & -32.6 & -8.4 & 3.9 & 0.036 & 2.139 \\
\hline 12.115 & 14.808 & Reversal & -48 & -5.4 & 2.7 & 0.236 & 15.056 \\
\hline 12.270 & 15.000 & Inflected & -48 & -6.3 & 2.7 & 0.084 & 3.807 \\
\hline 16 & 19.615 & Through & -3 & -16.2 & 16.6 & 0.124 & 5.460 \\
\hline
\end{tabular}

\section{Numerical simulations}

Results for the MA1400 and 50E robots are presented in Tables 2 and 3, respectively. In these tables, deflection is the amount the joint 9 tool-motion axis is tipped as depicted in Fig. 3 in Section 2, whereas deviation is the extremal $\theta_{7}$ along the adjusted path followed by the avoidance maneuver.

The mean calculation time over conditions in the two tables is $0.983 \mathrm{~ms}$ (Intel Core i5 $2.5 \mathrm{GHz}$, 64-bit OpenJDK 10 and Windows 10), separating into $0.038 \mathrm{~ms}$ to locate the singularity, $0.514 \mathrm{~ms}$ for avoidance planning, and $0.431 \mathrm{~ms}$ when executing the avoidance maneuver. After doubling the times 

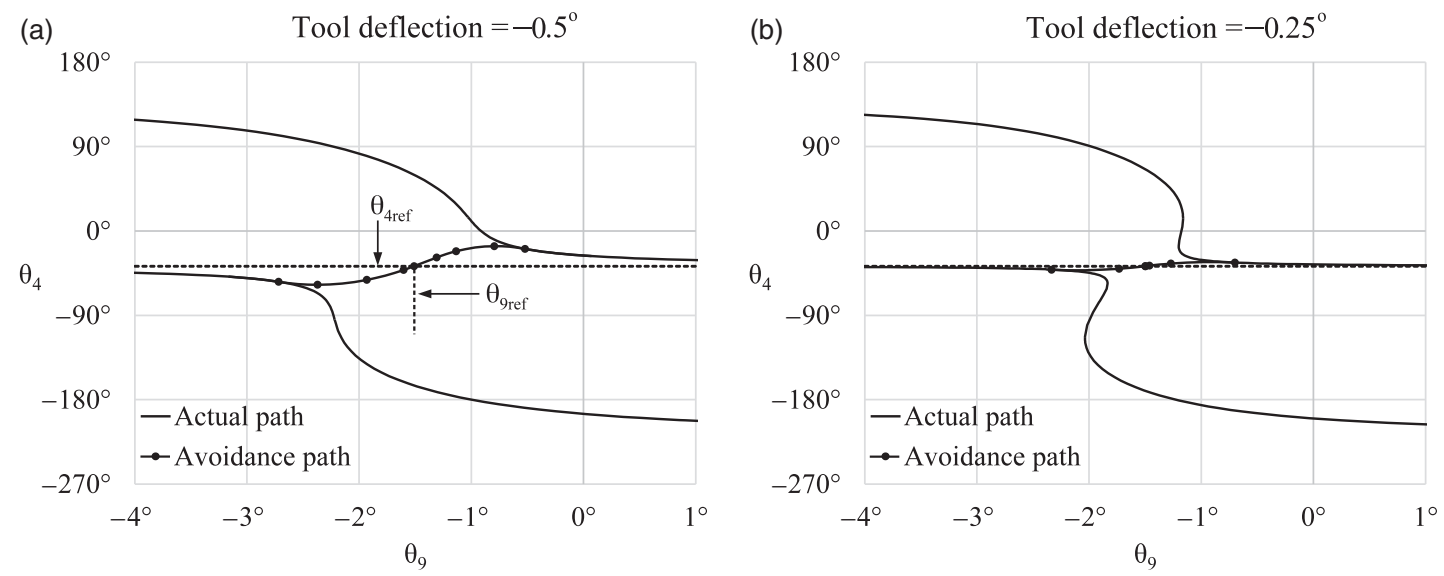

Figure 4. MA1400 robot path transition between through and 2 s-curves conditions.
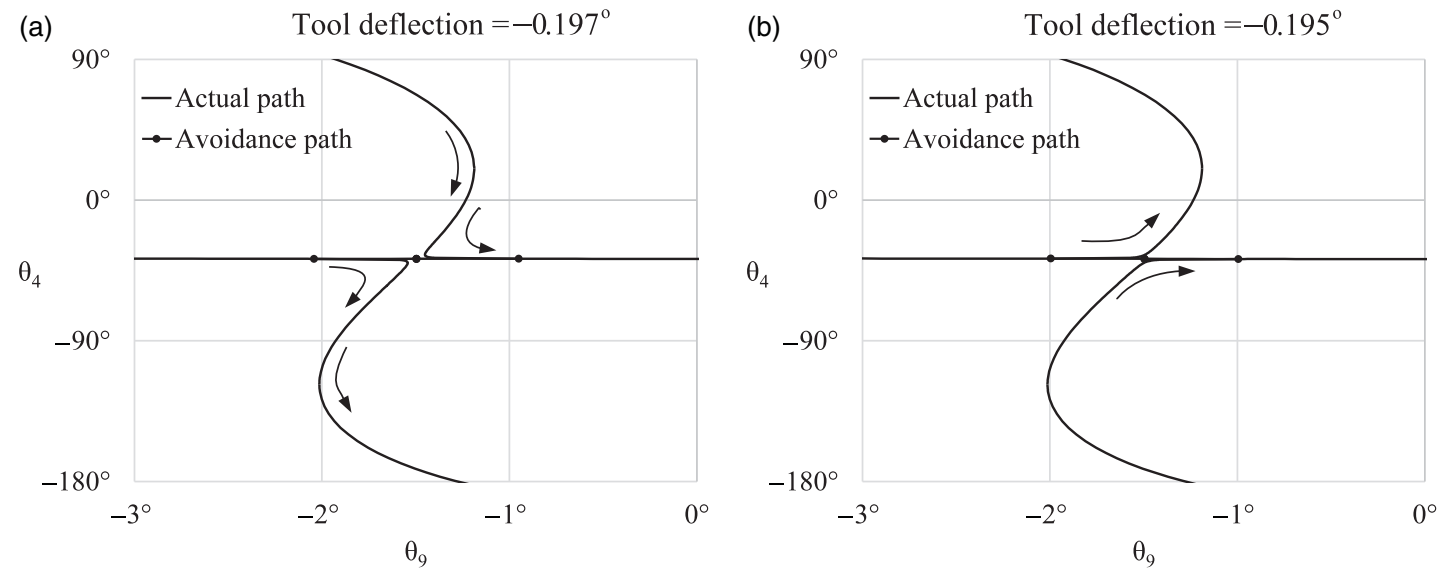

Figure 5. MA1400 robot initial path bifurcation between 2 s-curves and path reversal.

for portions conducted on only one side of the singularity for proper comparison, timings for the prior spherical-wrist method are [1] 0.030,0.174, and $0.246 \mathrm{~ms}$, calculated at $-8^{\circ}$ tool deflection. Comparing to the same large deflection, the new method applied to the MA1400 at $-8^{\circ}$ requires $0.044,0.696$, and $0.270 \mathrm{~ms}$. The path planning time for an offset wrist is hence seen to be substantially increased owing to locating singularities along the actual path, but the avoidance execution time is nearly the same. Having a worst-case overall time of $1.335 \mathrm{~ms}$, the offset-wrist procedures remain favorable for real-time use.

The purpose of the avoidance maneuver is to limit $C_{4} / C_{9}$, the ratio of rate on wrist joint 4 in relation to the tool traversal rate on joint 9 . Rate $C_{6}$ on joint 6 may exceed the $C_{4}$ limit by a modest factor, but many commercial robots have a higher rate limit for joint 6 . For a spherical wrist conducting a task in spherical space, limiting $C_{4} / C_{9}$ to \pm 6 generates approximately a $10^{\circ}$ exclusion cone for an actual path passing near the wrist singularity [1]. A high avoidance-maneuver rate limit $\left(C_{4} / C_{9}= \pm 48\right)$ along with a high acceleration limit (5000rad $/ \mathrm{s}^{2}$ at $C_{9}=1 \mathrm{rad} / \mathrm{s}$ ) is evaluated. The rate limit is subsequently reduced as needed to be below what is measured on the actual path, otherwise, the avoidance maneuver isn't needed. This accounts for lower $C_{4} / C_{9}$ limits at higher deflection angles in the tables. Otherwise, the higher rate limit is a more severe test of how close the initiation of avoidance can be to a singularity along the actual path.

The sequence of varying tool deflections in Figs. 4, 5 and 6 illustrates the evolution of conditions listed in the actual path column of Table 2. Displacement paths are depicted by plots of wrist angle $\theta_{4}$ 
(a)

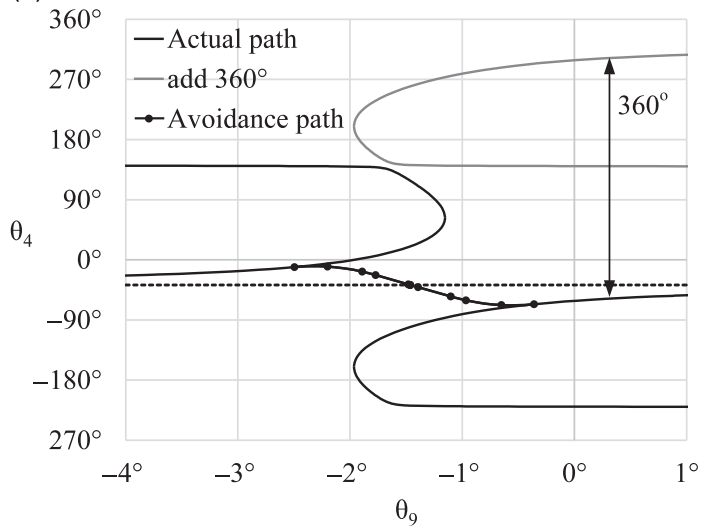

(b)

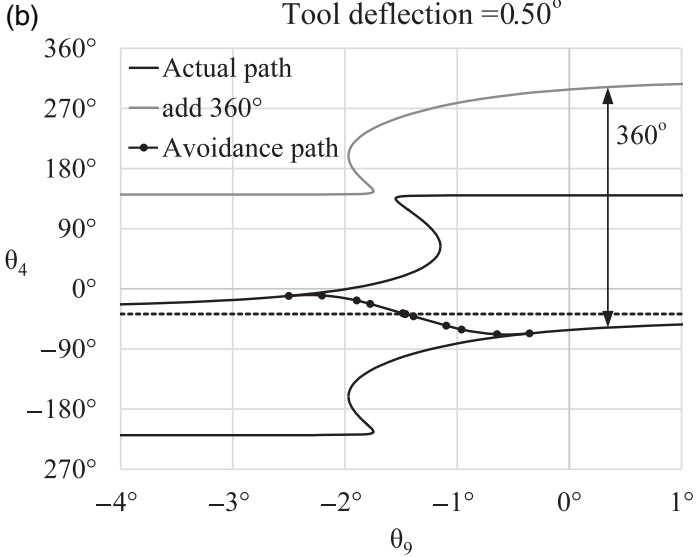

Figure 6. MA1400 robot final path bifurcation between reversal and 2 s-curves.

in relation to tool-traversal angle $\theta_{9}$. An actual path is where tool deviation $\theta_{7}=0$. The steep portion of such a path indicates a high rate on joint 4 for a constant rate of tool traversal on joint 9 . The point $\left(\theta_{9 \text { ref }}, \theta_{4 \text { ref }}\right)$ of least-maximum tool deviation $\theta_{7}$ is found according to Section 3.2. The dashed line for $\theta_{9 \text { ref }}$ contributes to a step-size bound on $\theta_{4}$ locating either a path inflection or an initial reversal by the method of Section 3.4. The search was restarted manually as required for plotting s-curves in Figs 4(b), 5(a) and 6(b). Planning and execution of paths that avoid high rates by allowing $\theta_{7}$ to vary are completely automated.

Figure 4(a) depicts the through condition showing two branches of the robot inverse-kinematic solution along paths allowing a continuous tool traversal rate. These branches are separated by a "flip" of joint 4 [4], exactly $180^{\circ}$ for a spherical wrist, and approximately this amount for an offset wrist. The through condition occurs on all paths passing nearby the singularity of a spherical wrist. A path avoiding the steep, high-rate portion of the actual path joins two branches with a shallower slope governed by a joint rate limit. The rounding of this slope to meet the actual path is governed by an acceleration limit. Starting at $\left(\theta_{9 \mathrm{ref}}, \theta_{4 \mathrm{ref}}\right)$, the procedures in Sections 3.3 and 3.4 work outward from that location to plan this avoidance maneuver. The ends of steps in the execution of the maneuver are marked in the plots.

Figure 4(b) depicts the $s$-curve condition occurring on two connected paths. It still allows a complete tool traversal, where the tool motion represented by joint 9 allowed to cross through zero rate, reverse, and then do this a second time to continue. The reversal condition occurring in Figs. 5(b) and 6(a) introduces an orientational exclusion zone between two isolated segments of the actual path. The robot is blocked from completing the tool traversal along the actual path, and it can only cross that zone when tool deviation is permitted on joint 7. An avoidance maneuver conducts that crossing. Each path reversal also exhibits a change in sign of $\theta_{5}$, meaning each isolated segment of the actual path passes through the notional offset-wrist singularity and changes branch as defined by Cheng et al. [4].

Tool deflections $-0.195^{\circ}$ and $0.49^{\circ}$ in Table 2 and Figs. 5(b) and 6(a) hence bound the small yet finite range of a turning-point singularity for the wrist of the MA1400 robot. The "bifurcated" condition in Tables 2 and 3 marks the transition to a fully developed turning point seen in Fig. 5(a) and (b). Figure 6(a) and (b) show the second bifurcation at the other end of the range of tool deflection exhibiting path reversal. This bifurcation occurs on the opposite side from where the avoidance maneuver meets the actual path. It is made more apparent by plotting the right-hand path segment after adding $360^{\circ}$. This gives identical displacement on revolute joint 4 because $360^{\circ}$ is a complete cycle of joint rotation.

Table 3 shows the 50E robot to switch between through and reversal paths without the transitional s-curve condition. The inflected condition noted in the table occurs on a reversal path. Its onset is when the tool deviation crosses below $12.27^{\circ}$, and an inflected reversal is shown for $12.2^{\circ}$ in Fig. 7 (a). Tracing the left-most curve in its tool-traversal direction, starting at the bottom, and continuing up to its abrupt 
(a)

Intercept paths

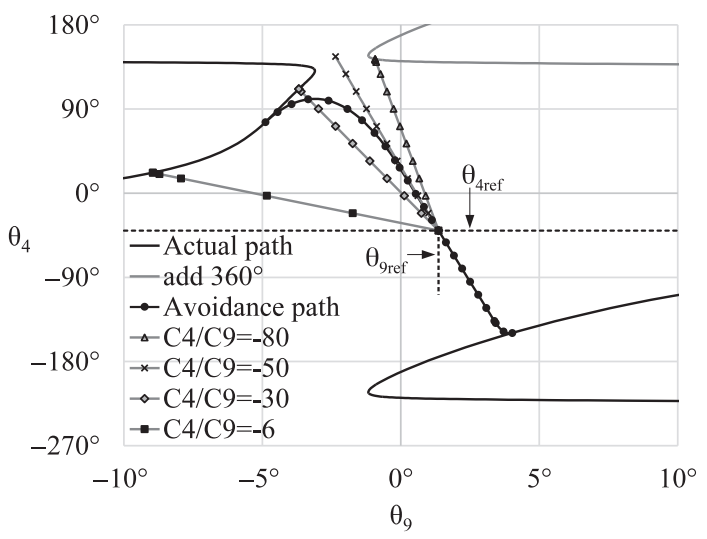

(b)

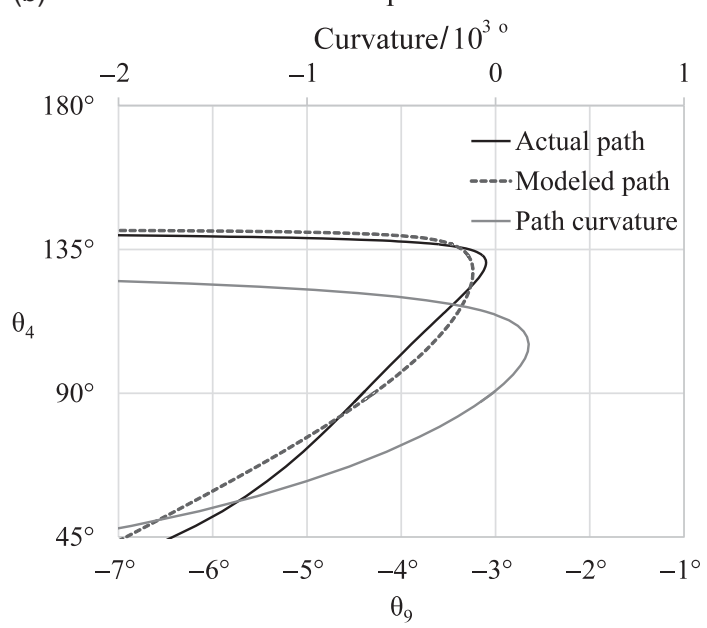

Figure 7. 50 E robot at $12.2^{\circ}$ tool deflection where (a) shows paths intercepting a path reversal that contains slope inflections (b) magnified view, where these inflections occur at the curvature zero crossings.

reversal near its top, it shows a pair of shallow inflections. This curve is magnified in Fig. 7(b). For this segment of the curve, $\theta_{9}$ is a single-valued function of $\theta_{4}$. Path curvature $d^{2} \theta_{9} / d \theta_{4}^{2}$ is shown on the same chart. The chart also shows the parametric model from Section 4.2 to approximate the actual path. The range of $\theta_{4}$ where the path is inflected is bounded by where curvature changes sign.

Tracing the right-hand path segment in Fig. 7(a) also required manual intervention. In taking steps of $\theta_{4}$ downward from $\theta_{4 \mathrm{ref}}$, the singularity locating procedure stopped short of the turning point where the closure error exceeded a threshold. This procedure does not need to reach the turning point, however, to bound the region of convergence limiting steps of $\theta_{9}$ along the actual path.

\subsection{Intercepting the actual path}

Section 3.3 raises the concern of reaching the actual path from the least-maximum point on the adjusted path. An intercept path starts at the point in the avoidance maneuver of maximum tool deviation and follows a line of constant $C_{4} / C_{9}$ slope as shown in Fig. 7(a). The actual-path region where $\theta_{9}$ as a function of $\theta_{4}$ has positive slope and negative curvature is intercepted by $C_{4} / C_{9}=-6$. This supplies a usable starting point for planning the avoidance maneuver. Ratio $C_{4} / C_{9}=-80$ intercepts the wrong segment but on a region of negative slope and positive curvature, so this intercept is rejected and $C_{4} / C_{9}$ is reduced by a factor of 2 before trying again. The intercept path at $C_{4} / C_{9}=-50$ does not intercept any branch, but method of Appendix B detects this to allow rejecting this intercept, updating $C_{4} / C_{9}$, and retrying. Ratio $C_{4} / C_{9}=-33$, however, intercepts a region between the inflections, where the slope and curvature are both positive. This intercept can also be rejected.

For all reported cases, rate ratio $C_{4} / C_{9}= \pm 6$ met three conditions on the sign of slope, curvature, and rate-of-change of curvature of the actual path at the point of intercept. This ratio gave usable pathplanning starting points. In instances found where these tests fail, the actual path could be followed backward to identify missed inflections.

\subsubsection{Kinematic model of path reversal}

Figures 8 and 9 are maps in stereographic projection of the surface of a sphere [46] used to visualize the articulation of a robotic wrist [1]. Alternatively, Milenkovic and Huang [25] use a joint-angle radialcoordinate plot not based on a map projection. In all such plots, the point of the axis direction vector of 
(a)

Wrist offset $=0$

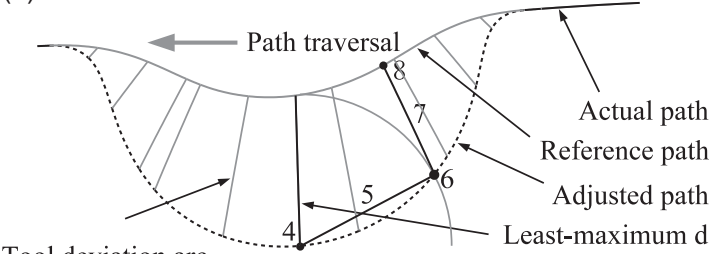

(b) Wrist offset $=0.170$

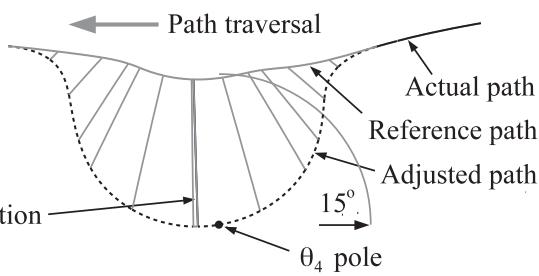

Figure 8. Singularity avoidance maneuver for $50 \mathrm{E}$ robot at $12.2^{\circ}$ tool deflection in stereographic projection (a) zero wrist offset and (b) offset $0.170 \mathrm{~m}$. The acceleration limit is set to $50 \mathrm{rad} / \mathrm{s}^{2}$, giving a smooth onset of the avoidance maneuver. The actual path divides into the reference path traced by the pole of joint 8 and the adjusted path traced by the pole of joint 6 when separated by a nonzero tool deviation arc 7.

(a)

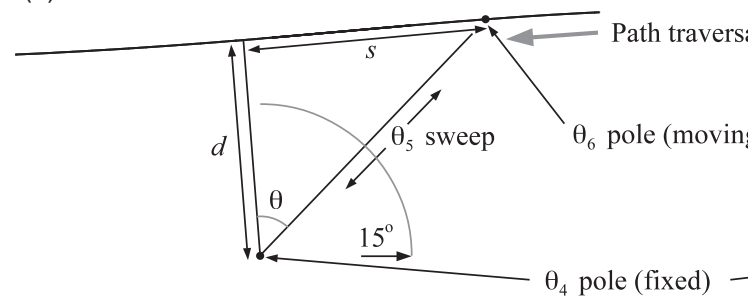

(b) Wrist offset $=0.170$

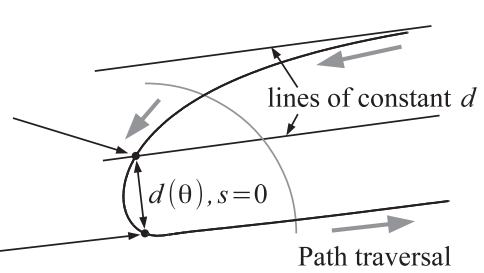

Figure 9. Actual path traced by joint 6 for the $50 E$ robot at $12.2^{\circ}$ tool deflection in stereographic projection of $(a)$ zero wrist offset and $(b)$ offset $0.170 \mathrm{~m}$. For clarity, only one of two actual-path branches of the offset wrist is shown.

a joint represents its pole. The maps in Figs. 8 and 9 are centered on the pole of joint 4 for the first joint in the robot wrist, marked by the fixed dot. As a consequence, they are polar plots where articulating joint 4 changes the angle and joint 5 changes radial distance to the dot for joint 6 .

The axis of joint 5 is located $90^{\circ}$ from joint 4 , separating its pole from that of joint 4 by a large distance outside the region of the map. The equator of joint 5 , however, is along a radial line of constant longitude in this map projection emanating from the pole of joint 4 . Varying $\theta_{5}$ sweeps the pole of joint 6 along that line. Actuating joint 6 can rotate another line passing through its pole in relation to the sweep induced by joint 5 , completing the change in body orientation effected by rotations about the three axes of the physical wrist. Setting $\theta_{5}=15^{\circ}$ and varying $\theta_{4}$ sweeps joint 6 along a non-equatorial latitude line, the quarter-circle shown on each map. Setting $\theta_{5}=0$ makes the poles of joints 4 and 6 coincident. Because the maps only represent orientation and thus cannot distinguish collinear from parallel axes, this condition marks the singular posture of a spherical wrist yet only the notional singularity of an offset wrist.

Figure 8 depicts the singularity avoidance maneuver for both a zero-offset spherical wrist and the offset wrist condition from Fig. 7. A forward tool traversal of increasing $\theta_{9}$ proceeds from right to left, which is reversed from Figs. 4, 5, 6, and 7. The path divides into reference and adjusted paths after the onset of tool deviation. The two paths are connected by the arc swept by the pole of joint 8 when articulating joint 7 . The least-maximum deviation occurs at the spherical wrist singularity. In an offset wrist, the least-maximum deviation can occur a small displacement away from the notional singularity.

The kinematic model is formulated by considering that when maintaining a target tool position, articulating an offset wrist also produces a translational displacement to be compensated by further articulation of the robot arm. This action of the arm, in turn, adds to the orientational change to be generated by the wrist. Hence even when their axis lines are parallel at that notional singularity, and 
unlike in the spherical wrist, joints 4 and 6 could still effect different orientational changes owing to such wrist-arm interaction.

Figure 9(b) shows a reversal in the actual path of an offset wrist. Even in the zero offset case where the actual path completes the traversal, the reference path in Fig. 8 shows a dip not seen in the Fig. 9(a) actual path. The axis of joint 8 gives the correct direction in relation to ground from which the tool deviates, but its change relative to joint 4 is attributable to arm/wrist coupling when articulating joints 7 and 8 , both from the offset of the physical wrist along with the separation between physical and virtual wrist centers.

Figure 9 shows angle $\theta=\theta_{4}-\theta_{4 \text { ref }}$, where $\theta_{4 \text { ref }}$ is the reference angle of joint 4 giving $-90^{\circ}<\theta<$ $90^{\circ}$. Path position $s$ is the similarly referenced value of joint 9 angle $\theta_{9}$ where $s=0$ and $\theta=0$ at the notional wrist singularity. Constant $d=\theta_{5}$ min is the minimum angle on joint 5 at close approach along the actual path. These relations applied to Fig. 9 give

$$
s=d \tan \theta
$$

This formula generates the through-path curve characteristic of a spherical wrist. The offset wrist at the tool deflection angle shown in Fig. 4(a) also shows such a curve. Treating $s$ as the independent variable, it also accounts for the rapid rotation about joint 4 along with its sudden onset when passing the wrist singularity at small $d$.

Considering the wrist in the 50E robot where the axis of joint 4 is offset from the intersection of joints 5 and 6 , rotation about joint 4 produces a circular translation about that intersection point. Joint 6 generates a similar translation in the MA1400 robot where the axis of joint 6 is offset from the other two wrist axes. The arm effects a change in orientation as it articulates to compensate for the translation generated by the wrist in either type of robot. Considering the robot and accompanying virtual joints to form a closed kinematic loop, this in turn changes the orientation of joint 9 with respect to a wristbased orientation frame. The resulting three degrees of orientational change may alter the offsets to $\theta_{4}$ and $\theta_{9}$ giving $\theta$ and $s$ along with the value of $d$. An approximation only considers $d$ changing with $\theta$ according to

$$
d(\theta)=d_{0}+d_{c} \cos \theta+d_{s} \sin \theta=d_{0}+d_{r} \cos \left(\theta-\theta_{r}\right)
$$

where either $d_{0}, d_{c}$, and $d_{s}$ or $d_{0}, d_{r}$, and $\theta_{r}$ form sets of three coefficients parameterizing the model. Over the course of a singularity encounter, $\theta_{4}$ and in turn $\theta$ vary over nearly a $180^{\circ}$ range, and the translation generated by the wrist follows nearly a complete semicircle. The robot arm is assumed to be sufficiently far from its singular positions that its orientational change is nearly proportional to the compensating translation of the arm along each of two Cartesian axes in the plane of the semicircle. Such sinusoidal displacement from articulating an offset wrist is observed by Trinh et al. [6].

This coupling of close-approach distance $d$ to angle $\theta$ replicates the behavior of an offset wrist undergoing a path reversal. Consider $\theta$ as the independent variable and $s$ as the dependent variable. Viewing Fig. 9(b) as $\theta$ increases from its initial value near $-90^{\circ}$, the path is traversed from right to left until the reduction in $d$ with increasing $\theta$ draws it back toward the joint 4 pole. When $d$ crosses through zero upon passage of a critical angle of $\theta$, the tool path crosses the joint 4 pole, also marked by wrist deflection angle $\theta_{5}$ changing sign. As $\theta$ increases toward its opposite asymptote at $+90^{\circ}$, the sign change of $d$ in Eq. (4) has reversed the path direction to proceed from left to right.

Expressing $\cos \theta, \sin \theta$, and $\tan \theta$ in terms of $t=\tan (\theta / 2)$, Eqs. (4) and (5) become

$$
0=s t^{4}+2\left(d_{0}-d_{c}\right) t^{3}+4 d_{s} t^{2}+2\left(d_{0}+d_{c}\right) t-s
$$

at path location $s$ derived from tool traversal angle $\theta_{9}$. This polynomial has no more than four real-valued roots in $t$, from which may be $\theta_{4}$ is derived. Tool deflections $-0.25^{\circ},-0.197^{\circ},-0.195^{\circ}, 0.49^{\circ}$, and $0.5^{\circ}$ from Figs. 4, 5, and 6 exhibit ranges of $\theta_{9}$ showing 4 solutions for $\theta_{4}$. Other ranges and figures show two solutions, and Fig. 7 has a range with zero solutions. A fourth-order polynomial does not allow either 1, 3 or more than 4 solutions, and there are no examples of these in Figs. 4, 5, 6, and 7. 
The modeled path plotted in Fig. 7(b) results from manual adjustment of parameters giving $\theta=\theta_{4}-$ $141.5^{\circ}+90^{\circ}, \theta_{9}=s-6.5^{\circ}$ and $d(\theta)=3.5^{\circ}\left(0.05+\cos \left(\theta+3.5^{\circ}\right)\right)$. For the model's range of $-90^{\circ}<$ $\theta<90^{\circ}, d(\theta)$ only crosses zero when $\theta$ reaches $89.4^{\circ}$. The model will switch from this reversal to a through path when the zero crossing is delayed beyond $90^{\circ}$. This accounts for a path bifurcation being sensitive to small parameter changes in the model.

Whereas the model also exhibits a path inflection where its curvature changes sign, this occurs over lower values of $\theta_{4}$ than the actual wrist in Fig. 7. The model is not able to reproduce the sharp corner occurring in the actual path either. This level of approximation nevertheless offers insight into how an offset wrist can undergo a path reversal.

Interestingly, the offset wrist on the Universal Robots UR robot is not expected to show path reversals. The UR has the axes of joints 2 and 3 in the arm along with joint 4 of the wrist in parallel. At the notional singularity where joint 5 is articulated to place joint 6 in parallel with 4, joints 2, 3, 4, and 6 form a planar four-bar linkage. The translational displacement resulting for articulating joint 4 can be compensated by joints 2 and 3 to allow joint 6 to rotate about its axis line fixed in space. The orientation change produced by the arm is in this way compensated by rotation of joint 4, only. Offset $d$ is therefore not changed as joint 4 articulates similarly to a spherical wrist. A video produced by the CoRo lab at ETS in Montreal [47] shows this compensation.

\section{Conclusions}

A serial robot with a nonspherical offset wrist has more complicated than anticipated paths when encountering its wrist singularity. Added path types over a spherical wrist include single (turning point) and double (s-curve) path reversals. These occur over a small yet finite range of passage near the notional offset-wrist singularity where its first and third joints become parallel. An approximate kinematic model accounting for arm/wrist interaction replicates a turning point along the robot path, giving a wrist orientation exclusion zone.

Extending the prior procedure formulated for a spherical wrist [1] required meeting multiple rather than a single stationarity condition to find an adjusted displacement path giving a least-maximum tool deviation. The joint-rate ratio $C_{4} / C_{9}$, however, needed to be limited when tracking outward from the least-maximum point to locate before-and-after singularity-encounter path segments at zero tool deviation. A rate ratio of $C_{4} / C_{9}= \pm 6$ was found effective for the offset-wrist robots considered.

Owing to the different singular conditions occurring away from the notional singularity of an offset wrist, new procedures were required to locate them on the actual path. The ability to distinguish between either a through-path or a turning-point singularity is an important advance over the prior method. This allows determining the minimum path length bridging either through paths or reversal paths of the offset wrist.

Conflicts of Interest. The author declares has none.

\section{References}

[1] P. Milenkovic, "Continuous path control for optimal wrist singularity avoidance in a serial robot," Mech. Mach. Theory 140, 809-824 (2019). doi: 10.1016/j.mechmachtheory.2019.05.004 0094-114X

[2] M. K. Özgören, "Kinematic analysis of a manipulator with its position and velocity related singular configurations," Mech. Mach. Theory 34(7), 1075-1101 (1999).

[3] B. Huang and V. Milenkovic, "Method to Avoid Singularity in a Robot Mechanism," U.S. Patent and Trademark Office patent US 4,716,350 (1987).

[4] S. K. Cheng, M. R. Jean, H. D. McGee, C. K. Tsai and D. Xiao, "Method of Controlling a Robot through a Singularity," U.S. Patent and Trademark Office patent US 6,845,295 (2005).

[5] R. I. Association, ANSI/RIA r15. 06 American National Standard for Industrial Robots and Robot Systems-Safety Requirements (Robotics Industries Association, Ann Arbor, 1999). 
[6] C. Trinh, D. Zlatanov, M. Zoppi and R. Molfino, "A Geometrical Approach to the Inverse Kinematics of 6r Serial Robots with Offset Wrists," ASME 2015 International Design Engineering Technical Conferences and Computers and Information in Engineering Conference (2015) pp. 1-10.

[7] M.-K. Wu, Y.-S. Kung, F.-C. Lee and W.-C. Chen, "Inverse Kinematics of Robot Manipulators with Offset Wrist," 2015 International Conference on Advanced Robotics and Intelligent Systems (ARIS) (2015) pp. 1-6.

[8] D. Corinaldi, M. Callegari and J. Angeles, "Singularity-free path-planning of dexterous pointing tasks for a class of spherical parallel mechanisms," Mech. Mach. Theory 128, 47-57 (2018). doi: 10.1016/j.mechmachtheory.2018.05.006 0094-114X

[9] T. Sun, D. Liang and Y. Song, "Singular-perturbation-based nonlinear hybrid control of redundant parallel robot," IEEE Trans. Ind. Electron. 65(4), 3326-3336 (2018).

[10] M. K. Ozgoren, "Kinematic and kinetostatic analysis of parallel manipulators with emphasis on position, motion, and actuation singularities," Robotica 37(4), 599-625 (2019).

[11] Y. Huang, Y. S. Yong, R. Chiba, T. Arai, T. Ueyama and J. Ota, "Kinematic control with singularity avoidance for teachingplayback robot manipulator system," IEEE Trans. Automat. Sci. Eng. 13(2), 729-742 (2016).

[12] C. W. Wampler, "Manipulator inverse kinematic solutions based on vector formulations and damped least-squares methods," IEEE Trans. Syst. Man Cybernet. 16(1), 93-101 (1986).

[13] Y. Nakamura and H. Hanafusa, "Inverse kinematic solutions with singularity robustness for robot manipulator control," ASME J. Dyn. Syst. Meas. Cont. 108(3), 163-171 (1986).

[14] L. W. Tsai, Robot Analysis: The Mechanics of Serial and Parallel Manipulators (John Wiley and Sons, New York, 1999).

[15] S. Chiaverini, B. Siciliano and O. Egeland, "Review of the damped least-squares inverse kinematics with experiments on an industrial robot manipulator," IEEE Trans. Cont. Syst. Technol. 2(2), 123-134 (1994).

[16] A. A. Maciejewski and C. A. Klein, "Numerical filtering for the operation of robotic manipulators through kinematically singular configurations," J. Robot. Syst. 5(6), 527-552 (1988).

[17] X. Wang, D. Zhang, C. Zhao, H. Zhang and H. Yan, "Singularity analysis and treatment for a 7r 6-dof painting robot with non-spherical wrist," Mech. Mach. Theory 126, 92-107 (2018). doi: 10.1016/j.mechmachtheory.2018.03.018 0094-114X

[18] Y. Wan, Y. Kou and X. Liang, "Closed-Loop Inverse Kinematic Analysis of Redundant Manipulators with Joint Limits," International Conference on Mechanical Design (2017) pp. 1241-1255.

[19] Y. Nakamura, Advanced Robotics: Redundancy and Optimization (Addison-Wesley Longman Publishing Co., Inc., Reading, Massachusetts, 1991).

[20] D.-E. Kim, D.-J. Park, J.-H. Park and J.-M. Lee, "Collision and Singularity Avoidance Path Planning of 6-DOF Dual-Arm Manipulator," International Conference on Intelligent Robotics and Applications (2018) pp. 195-207.

[21] V. Chembuly and H. K. Voruganti, "An Optimization Based Inverse Kinematics of Redundant Robots Avoiding Obstacles and Singularities," Proceedings of the Advances in Robotics (2017) p. 24.

[22] S. R. Buss and J.-S. Kim, "Selectively damped least squares for inverse kinematics," J. Graph. GPU, Game Tools 10(3), 37-49 (2005).

[23] S. Chiaverini and O. Egeland, "A Solution to the Singularity Problem for Six-Joint Manipulators," IEEE International Conference on Robotics and Automation, vol. 1 (1990) pp. 644-649.

[24] D. Oetomo and M. H. Ang, Jr., "Singularity robust algorithm in serial manipulators," Robot. Comput. Integr. Manufact. 25, 122-134 (2009). doi: 10.1016/j.rcim.2007.09.007

[25] V. Milenkovic and B. Huang, "Development On an Algorithm Negotiating Wrist Singularities," Robot 11/17th ISIR (1987) pp. 13-1-13-6.

[26] E. W. Aboaf and R. P. Paul, "Living with the Singularity of Robot Wrists," IEEE International Conference on Robotics and Automation (1987) pp. 1713-1717.

[27] P. Milenkovic, "Effect of the Coordinate Frame on High-Order Expansion of Serial-Chain Displacement," Proceedings of the Institution of Mechanical Engineers, Part K: Journal of Multi-body Dynamics (2019) pp. 840-855.

[28] J. Kieffer, "Manipulator inverse kinematics for untimed end-effector trajectories with ordinary singularities," Int. J. Robot. Res. 11(3), 225-237 (1992).

[29] X. Luo, S. Li, S. Liu and G. Liu, "An optimal trajectory planning method for path tracking of industrial robots," Robotica 37(3), 502-520 (2019).

[30] H. Tourajizadeh and O. Gholami, "Optimal control and path planning of a 3PRS robot using indirect variation algorithm," Robotica 38(5), 903-924 (2020).

[31] M. R. Vezvari, A. Nikoobin and A. Ghoddosian, "Perfect torque compensation of planar 5R parallel robot in point-to-point motions, optimal control approach," Robotica, 39, 1-18 (2020).

[32] S. Mansouri, M. J. Sadigh and M. Fazeli, "A computationally efficient algorithm to find time-optimal trajectory of redundantly actuated robots moving on a specified path," Robotica 37(1), 62-79 (2019).

[33] A. Griewank and A. Walther, "On the Efficient Generation of Taylor Expansions for DAE Solutions by Automatic Differentiation," In: Applied Parallel Computing. State of the Art in Scientific Computing. PARA 2004 (J. Dongarra, K. Madsen and J. Wasniewski, eds.) (Springer, Berlin, Heidelberg, 2006) pp. 1089-1098.

[34] P. Milenkovic, "Series solution for finite displacement of single-loop spatial linkages," ASME J. Mech. Robot. 4(2), 021016 (2012). 
[35] R. Di Gregorio, "Acceleration and higher-order analyses solved by extending the superposition principle: The incipient motion technique," Mech. Mach. Theory 153, 103953 (2020). doi: 10.1016/j.mechmachtheory.2020.1039530094-114X

[36] A. A. Hassan, M. El-Habrouk and S. Deghedie, "Inverse kinematics of redundant manipulators formulated as quadratic programming optimization problem solved using recurrent neural networks: A review," Robotica 38(8), 1495-1512 (2020).

[37] G. Huber and D. Wollherr, "An online trajectory generator on se (3) for human-robot collaboration," Robotica 38(10), 1756-1777 (2020).

[38] S. Blanes, F. Casas, J.-A. Oteo and J. Ros, "The magnus expansion and some of its applications," Phys. Rep. 470(5-6), 151-238 (2009).

[39] R. Achilles and A. Bonfiglioli, "The early proofs of the theorem of campbell, baker, hausdorff, and dynkin," Arch. His. Exact Sci. 66(3), 295-358 (2012).

[40] Z.-H. Kang, C.-A. Cheng and H.-P. Huang, "A singularity handling algorithm based on operational space control for sixdegree-of-freedom anthropomorphic manipulators," Int. J. Adv. Robot. Syst. 16(3), 1729881419858910 (2019).

[41] J. E. Lloyd and V. Hayward, "Singularity-robust trajectory generation," Int. J. Robot. Res. 20(1), 38-56 (2001).

[42] E. D. Pohl and H. Lipkin, "A New Method of Robotic Rate Control near Singularities," IEEE International Conference on Robotics and Automation (1991) pp. 1708-1713.

[43] J. E. Lloyd, "Desingularization of nonredundant serial manipulator trajectories using puiseux series," IEEE Trans. Robot. Automat. 14(4), 590-600 (1998).

[44] "Motoman-ma1400 instructions" (n.d.).

[45] A. Pashkevich, "Real-time inverse kinematics for robots with offset and reduced wrist," Cont. Eng. Pract. 5(10), 1443-1450 (1997).

[46] J. P. Snyder, Map Projections: A Working Manual (US Government Printing Office, 1987).

[47] What Are the Singularities of a Typical Collaborative Robot (Cobot) (École de Technologie Supérieure, 2018).

[48] P. Milenkovic, "Solution of the forward dynamics of a single-loop linkage using power series," ASME J. Dyn. Syst. Meas. Cont. 133(6), 061002 (2011).

[49] P. Milenkovic, "Projective constraint stabilization for a power series forward dynamics solver," ASME J. Dyn. Syst. Meas. Cont. 135(3), 031004 (2013).

[50] R. Featherstone, Rigid Body Dynamics Algorithms (Springer, New York, 2008).

[51] R. Featherstone, "Plucker Basis Vectors," Proceedings 2006 IEEE International Conference on Robotics and Automation (2006) pp. 1892-1897.

[52] C. B. Garcia and W. I. Zangwill, Pathways to Solutions, Fixed Points, and Equilibria (Prentice-Hall, Englewood Cliffs, New Jersey, 1981).

[53] S. Ambike and J. P. Schmiedeler, "A methodology for implementing the curvature theory approach to path tracking with planar robots," Mech. Mach. Theory 43(10), 1225-1235 (2008).

[54] S. Ambike, J. P. Schmiedeler and M. M. Stanisic, "Trajectory tracking via independent solutions to the geometric and temporal tracking subproblems," ASME J. Mech. Robot. 3(2), 021008 (2011).

[55] W. G. Bickley, “1587. An extension of newton's formula for approximating to the roots of equations," Math. Gazette 26(269), 102-104 (1942).

[56] W. H. Press, B. P. Flannery, S. A. Teukolsky and W. T. Vetterling, Numerical Recipes in Pascal (Cambridge University Press, New York, 1989).

\section{Appendix A}

The guided displacement path-following method [1] is extended to multiple guiding coefficients as required to optimize the avoidance path for an offset wrist. Minor optimizations omitting zero terms in summations are not shown.

The robot joint numbers start at 1 at the robot base. To keep the same notation used in prior work [27, 34, 48], the grounded base is numbered link 1 , which is connected to initial moving link 2 in the robot by joint 1 . A nonredundant serial robot is extended with a virtual joint representing tool motion that closes a kinematic chain. This loop allows solving the robot inverse kinematics giving the desired tool displacement using a path-following algorithm[49]. The guided displacement method can supply joint rates allowing a similar solution for a redundant robot. The series expansion of a serial robot is restated in what follows, the derivation of the guided displacement from these equations is extended to multiple coefficients, and finally, such guiding is directed by one or more virtual displacements occurring along an actual displacement path.

A revolute joint has screw $T_{j}=\left(\boldsymbol{\omega}_{j} ; \mathbf{v}_{0 j}=\mathbf{r}_{j} \times \omega_{j}\right)$ constructed from a pair of three-element vectors: unit length $\boldsymbol{\omega}_{j}$ gives the joint axis direction, $\mathbf{r}_{j}$ selects a point on the joint axis line, and $\times$ denotes the vector cross product [50, 51]. The instantaneous screw or twist $T_{C i}$ of link $i$ in the above numbering scheme is

$$
T_{C i}=\sum_{j=1}^{i-1} C_{j} T_{j}
$$


where $C_{j}$ is the rate of joint $j$ having joint screw $T_{j}$. Apart from joints connected to ground at each end of a chain, the joint screw in turn varies according to the differential equation

$$
\dot{T}_{i}=T_{C i} \times T_{i}
$$

for $T_{C i}$ the instantaneous screw imparted on the link immediately preceding the joint in the designated numbering scheme and Lie product $\mathbf{x}($ boldface $\times)$. For these variables varying with time and having series expansions

$$
\begin{gathered}
T_{j}=T_{j}(t)=T_{j}[0]+T_{j}[1] t+T_{j}[2] t^{2}+\cdots, \\
C_{j}=C_{j}(t)=C_{j}[0]+C_{j}[1] t+C_{j}[2] t^{2}+\cdots, \\
T_{C i}=T_{C i}(t)=T_{C i}[0]+T_{C i}[1] t+T_{C i}[2] t^{2}+\cdots
\end{gathered}
$$

Equation (A1) relates the series coefficients of these variables by

$$
\begin{aligned}
T_{C i}[k] & =\sum_{j=1}^{i-1} C_{j}[k] T_{j}+T_{B C i}[k] \\
T_{B C i}[k] & =\sum_{j=1}^{i-1} \sum_{m=0}^{k} \tilde{C}_{j}[m] T_{j}[k-m]=\sum_{j=1}^{i-1} \tilde{C}_{j}[k] * T_{j}[k]
\end{aligned}
$$

where initial joint screws $T_{j}=T_{j}[0]=T_{j}(0)$ and $\tilde{C}_{j}[m]=C_{j}[m]$ apart from $\tilde{C}_{j}[k]=0$. Operator $*$ denotes discrete convolution expressed by the inner summation. For link $i=n+1$ closing the chain, the loop closure constraint $T_{C n+1}(t)=0$ is expressed

$$
A C[k]+T_{B C n+1}[k]=0
$$

where the columns of matrix $A$ are the initial joint screws $T_{i}$. Solving for rate vector $C[k]$ comprised of elements $C_{i}[k]$ subsequently allows updating $T_{C i}[k]$ from $T_{B C i}[k]$ in Eq. (A3). Typically, a solution is found by designating a nonredundant subset of coefficients $C_{i}$ as passive joint rates, whereas the remaining coefficients are designated as independent variables controlling the active joints. Setting one or more of these independent variables to zero locks those joints. When the calculated loop closure error [49] accumulates at the end of a path-following step, a nonzero $T_{B C n+1}[0]$ is applied to the next step in a combined predictor-corrector procedure $[27,52,53]$.

Equation (A2) tracks the joint screws over a finite displacement giving

$$
T_{i}[k+1]=\frac{1}{k+1} \dot{T}_{i}[k]=\frac{1}{k+1} T_{C i}[k] *_{\times} T_{i}[k]
$$

that in turn determines $C[k+1]$. Operator $*_{\mathbf{x}}$ denotes discrete convolution over the Lie product $\mathbf{x}$.

The guided displacement method supplies additional independent variables to Eq. (A4) needed to meet constraining conditions. The joint-rate vector is separated into terms

$$
C[k]=C_{\gamma} \gamma[k]+C_{\beta}[k]
$$

where $\gamma[k]$ is the guiding coefficient $-\gamma$ denotes "guiding" and $\beta$ "baseline." In the prior single-guiding method, $C_{\gamma}$ is the column vector of joint-rate values $\left[C_{\gamma_{1}} \cdots C_{\gamma_{n}}\right]^{T}$ for $n$ joints and $\gamma[k]$ is a scalar [21]. Multiple guiding coefficients are elements of column vector $\gamma[k]=\left[\gamma_{1}[k] \cdots \gamma_{\nu}[k]\right]^{T}$. Row vectors $C_{\gamma i}=\left[C_{\gamma i, 1} \cdots C_{\gamma i, v}\right]$ for $1 \leq i \leq n$ make up the $n \times v$ matrix $C_{\gamma}$ for number of guiding coefficients $v$. Hence product $C_{\gamma} \gamma[k]$ remains a column vector under either single or multiple guiding.

Equation (A4) is satisfied for all $\gamma[k]$ upon substituting $C[k]$ from Eq. (A6) into Eq. (A4) giving

$$
A C_{\gamma}=0, \quad A C_{\beta}[k]+T_{B C n+1}[k]=0
$$

The one or more columns of $C_{\gamma}$ giving homogeneous solutions to the linear system with matrix $A$ are calculated once at the start of a series expansion. Subsequently at each index $k$, scalar or vector $\gamma[k]$ weights this collection of homogenous solutions to meet one or more side conditions. The resulting value of $C[k]$ then updates the value of $T_{B C n+1}[k+1]$ to allow solving Eq. (A7) for $C_{\beta}[k+1]$.

Substituting Eq. (A6) into (A3) gives the influence of $\gamma[k]$ on the link instantaneous screws

$$
\begin{aligned}
T_{C i}[k] & =T_{\gamma C i} \gamma[k]+T_{\beta C i}[k], \text { where } \\
T_{\gamma C i} & =\sum_{j=1}^{i-1} T_{j} C_{\gamma j}, \quad T_{\beta C i}[k]=\sum_{j=1}^{i-1} C_{\beta j}[k] T_{j}+T_{B C i}[k]
\end{aligned}
$$


Substituting this expression of the instantaneous screw into Eq. (A5) does the same for the joint screw

$$
\begin{aligned}
T_{i}[k+1] & =\frac{1}{k+1} T_{\gamma i \gamma}[k]+T_{\beta i}[k+1], \text { where } \\
T_{\gamma i, r} & =T_{\gamma C i, r} \times T_{i} \text { for } 1 \leq r \leq v, \quad T_{\beta i}[k+1]=\frac{1}{k+1} \tilde{T}_{C i}[k] *_{x} T_{i}[k]
\end{aligned}
$$

and where $\tilde{T}_{C i}[m]=T_{C i}[m], m<k$ and $\tilde{T}_{C i}[k]=T_{\beta C i}[k]$. As with $C_{\gamma i}, T_{\gamma C i}=\left[T_{\gamma C i, 1} \cdots T_{\gamma C i, v}\right]$ and $T_{\gamma i}=\left[T_{\gamma i, 1} \cdots T_{\gamma i, v}\right]$ are right-multiplied by scalar or column vector $\gamma[k]$ for one or more guiding coefficients. For Lie product $\mathbf{x}, T_{\gamma i, r}=T_{\gamma} C i, r \mathbf{x} T_{i}$ applies over the range of guiding-coefficient index $r$. As with $C_{\gamma}$, the one or more columns of $T_{\gamma} \mathrm{Ci}_{i}$ and $T_{\gamma i}$ to be multiplied by the one or more guiding coefficients are calculated once at the start of a series expansion. In this way, Eqs. (A6)-(A9) of the scalar guiding coefficient formulation [1] have been extended to the vector case allowing multiple guiding coefficients.

The guiding coefficients $\gamma[k]$ are calculated to meet constraints on the series coefficients of one or more joint-rate vectors $C_{V}(t)$ comprised of elements $C_{V i}(t)=\delta \theta_{i}(t) / \delta x$. These elements represent a virtual displacement $\delta \theta_{i}(t)$ of joint angle $\theta_{i}$ at time $t$ with respect to variation $\delta x$ in parameter $x$. This is an infinitesimal displacement satisfying $A(t) C_{V}(t)=0$ at all times $t$ along the actual, finite-displacement path. The trajectories of the joint screws $T_{i}(t)$ making up the columns of $A(t)$, however, are determined as before from the joint rates $C(t)$ on the actual. Because the virtual displacement $C_{V}(t)$ is a property of the mechanism posture having angles $\theta(t)$ determining those joint screws through the forward kinematic constraint, instantaneous rate vector $C_{V}$ can represent a constraint on joint-angle vector $\theta$.

The influence of $\gamma[k]$ on the joint screws $T_{i}[k+1]$ from Eq. (A9) will be applied to $A(t)$ in the solution of $A(t) C_{V}(t)=0$. For simplicity of the following formulas, only a single virtual displacement $C_{V}(t)$ is considered. This generalizes to multiple virtual displacements $C_{V, r}(t)$, with each value of index $r$ selecting one of those virtual displacements. Initial series coefficient $C_{V}[0]=C_{V}(0)$ is determined by solving $A C_{V}[0]=0$ for one or more active joint rates among the elements of $C_{V}$ driving the virtual displacement; $C_{V}[0]$ is in this way determined by the initial joint angles of the robot along its actual displacement path. To see how successive coefficients $C_{V}[k+1]$ for $k \geq 0$ are linear in the elements of the guiding vector $\gamma[k]$ adding a constant offset,

$$
A C_{V}[k+1]+\sum_{i=1}^{n} \tilde{C}_{V i}[k+1] * T_{i}[k+1]=0
$$

where $\tilde{C}_{V i}[m]=C_{V i}[m]$ apart from $\tilde{C}_{V i}[k+1]=0$. According to Eq. (A9), scalar $\gamma[k]$ influences joint screw coefficients $T_{i}[k+1]$, which in turn determines $C_{V}[k+1]$ by Eq. (A10). Substituting

$$
C_{V}[k+1]=\frac{1}{k+1} C_{\gamma V \gamma}[k]+C_{\beta V}[k+1]
$$

together with Eq. (A9) into Eq. (A10) and then grouping by dependence on $\gamma[k]$ gives

$$
\begin{aligned}
& A C_{\gamma V}+\sum_{i=1}^{n} C_{V i}[0] T_{\gamma i}=0, \\
& A C_{\beta V}[k+1]+\sum_{i=1}^{n} \tilde{C}_{V i}[k+1] * \tilde{T}_{i}[k+1]=0
\end{aligned}
$$

Constant $C_{\gamma V}$ is an $n \times v$ matrix as is $C_{\gamma}$ that is also solved once at the start of a series expansion whereas $C_{\beta V}[k+1]$ is solved at each index $k \geq 0$. Each $\gamma[k]$ controls the next series coefficient $C_{V}[k+1]$ by way of Eq. (A11). In a single-guiding example where $C_{V 7}(0)=C_{V 7}[0] \neq 0, C_{V 7}(t)$ may be placed on a linear path restoring it to zero at $t=1$ by setting $\gamma[0]$ to make $C_{V 7}[1]=-C_{V 7}[0]$, with $\gamma[k]$ chosen to make $C_{V 7}[k+1]=0$ for $k \geq 1$. By correcting in this way for any $C_{V 7}(t) \neq 0$ at the start of each successive step that may result from predictor error in the preceding step, the side condition $C_{V 7}(t)=0$ is "achieved and maintained" along an actual displacement path.

In the instance of multiple side conditions of this form, Eqs. (A11) and (A12) will update series coefficients for the multiple instances of virtual-displacement joint-rate vectors $C_{V, r}(t)$ with initial coefficient $C_{V, r}$ [0], each of which having a separate matrix $C_{\gamma V, r}$ calculated once at the start of each series expansion along with its own baseline series coefficients $C_{\beta V, r}[k+1]$. This collection of virtual displacements, however, will share a single series of guiding coefficient vectors $\gamma[k]$.

In the multi-guiding solution to the problem posed in Section 3.2 having passive joints 1, 2, 3, 7, 8, and 9 with guiding joints 4, 5, and 6 and virtual displacement active joints 4, 5, and $6 \mathrm{p}, A C_{\gamma}=0$ is solved for the three columns of $C_{\gamma}$ and Eq. (A12) is solved for the three instances of $C_{\gamma V}$ designated $C_{\gamma V, r}$ for $1 \leq r \leq v=3$. When $C_{V, r}(0)=C_{V, r}[0] \neq 0$, the collection of virtual displacements $C_{V, r}(t)$ for $1 \leq r \leq v=3$ may be placed on linear paths restoring them to zero at $t=1$ by setting 3-vector $\gamma[0]$ to make each $C_{V, r}[1]=-C_{V, r}[0]$, with 3-vector $\gamma[k]$ chosen to make each $C_{V, r}[k+1]=0$ for $k \geq 1$. With the single selection of passive joint 7 in this example from each $C_{V, r}(t)$, Eq. (A11) applied to each virtual displacement vector $1 \leq r \leq v=3$ therefore generates a $3 \times 3$ system of linear equations solvable for the three elements of $\gamma[k]$.

Algorithm: Given the initial joint screws $T_{i}$ comprising matrix $A$, solve for constants $C_{\gamma}, T_{\gamma C i}, T_{\gamma i}$ along with the one or more vectors $C_{V}[0]$ and matrices $C_{\gamma V}$ defined above. For each index starting at $k=0$, perform the following steps. Evaluate Eqs. 
(a)

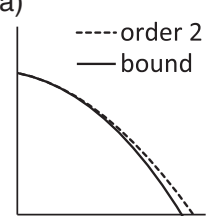

(c)

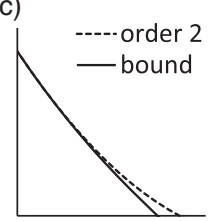

(b)

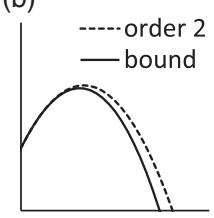

(d)

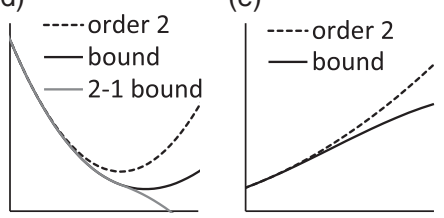

Figure 10. Cases of quadratic functions $f_{2}(x)$ (dashed lines) and their lower-bound polynomials $\breve{f}_{p}(x)$ (solid lines).

(A7)-(A9) for $C_{\beta}[k]$ and $T_{\beta i}[k+1]$ followed by Eq. (A12) for one or more coefficients $C_{\beta V}[k+1]$. Solve Eq. (A11) for $\gamma[k]$ meeting conditions on the one or more virtual displacement coefficients $C_{V}[k+1]$. Use this vector $\gamma[k]$ in Eqs. (A6), (A8), and (A9) to update $C[k], T_{C i}[k]$ and $T_{i}[k+1]$ on the actual displacement path.

\section{Appendix B}

Path-planning stages described in Section 3 require articulating the robot to bring a targeted joint angle, its rate or a rate derivative (angular acceleration) to zero. Prior procedures [1] displaced an active joint to make a coarse adjustment to the targeted quantity of a passive joint. The fine adjustment exchanges the roles of active and passive between the two joints, which allows directly declining a targeted joint angle to zero. In the case of a joint rate, the guided displacement method of Appendix A declines the ratio of its rate with that of another joint (a speed ratio [54]) to zero. Finding an accurate zero of a rate derivative is important for an offset wrist. The difference between this location and the notional singularity of the offset wrist can affect the convergence of path-following steps along with the starting and ending points of the maneuver bridging a path reversal. Applying a guided displacement to a rate derivative would require extending the complicated method of Appendix A to second order. Even in the case of an angle or rate, this is a reversion of series that introduces new concerns regarding series convergence.

A reversion of series expands the inverse function $x=g(y)$ from the coefficients of $y=f(x)$, exchanging the roles of independent and dependent variable. Inverse function $g$ is singular, however, at the pair of values $x, y$ where the forward function has an inflection point. The Newton-Raphson method finding a zero of $f(x)$ from its value and its derivative at a starting point $x_{1}$ may be regarded as a low-order reversion [55]; its iterations can diverge wildly near inflection $f^{\prime}\left(x_{1}\right)=0$ [56].

With a kinematic loop that admits a high-order power series expansion of the coupled Eqs. (A1) and (A2), a high-order guided displacement method potentially allows solving for a zero of a speed ratio over a large angle displacement of the guiding joint in one update step. Unfortunately, inflections of $f(x)$ giving the speed ratio as a function of a joint angle make $g(y)$ singular. Even if that singularity is some distance from the zero in question, a high-order series converges slowly or even diverges if the step size is large in relation to the distance to the inflection singularity. Such remains the case even when taking a step in the opposite direction. Along with the need to extend Appendix A to the rate derivative, $f(x)$ having an inflection on the scale of the distance to its zero motivates deriving an alternative.

Consider expanding $f(x)$ to high order, treating the truncated series as coefficients of polynomial $f_{p}(x)$, and solving for its first real root. This process is heuristic inasmuch as this polynomial is not $f(x)$. A high-order series truncation, however, gives small kinematic closure error when its steps are restricted according to the limits described in Section 3.4. Taking a final step that is a fraction $\rho$ of that limit makes the error of an order $p$ expansion even smaller by $O\left(\rho^{p+1}\right)$. The problem then is one of finding the first real root $x_{0}>0$, if any, of a polynomial. Newton-Raphson iterations are of low calculation cost when applied to a polynomial relative to function evaluations of the kinematic problem. Those iterations are prevented from overshooting the root by applying them to a bounding polynomial. The procedure used for course adjustments [1] is improved by considering all cases of the quadratic portion of this polynomial to ensure it contains one real root. The following also allows dispensing with reversion of series for the final, fine adjustment at minor increase in calculation cost.

The approximating polynomial $f_{p}(x)=f_{p}[0]+f_{p}[1] x+f_{p}[2] x^{2}+\cdots+f_{p}[p] x^{p}$ is normalized so that $f_{p}[0]=f_{p}(0) \geq 0$ without changing its roots. If $f_{p}(0) \equiv 0$, the first root is already located. The lower-bound polynomial $\breve{f}_{p}(x)$ retains the quadratic coefficients $f_{p}[0], f_{p}[1], f_{p}[2]$ and sets all positive coefficients $f_{p}[i]$ for $2<i \leq p$ to zero. This guarantees that $\breve{f}_{p}(x) \leq f_{p}(x)$ for positive $x$ and that its first real root is a lower bound on the roots of $f_{p}(x)$. Function $\breve{f}_{3: p}(x)$ leaves out the quadratic terms of $\breve{f}_{p}(x)$, ensuring $\breve{f}_{3: p}(x) \leq 0$ for $x \geq 0$ making it either constant or monotone down.

Considering the relationships between its order 2 quadratic part and the complete bounding polynomial in Fig. 10, case (a) has a quadratic of initial negative slope and curvature, whereas case (c) has negative slope, positive curvature, and its first real 
root on the positive real line. Both quadratics are monotone down, to which is added a nonpositive monotone function $\breve{f}_{3: p}(x)$, establishing $\breve{f}_{p}(x)$ to be monotone down in the interval up to the root of the quadratic. The bounding polynomial $\breve{f}_{p}(x)$ therefore has one real root between zero and this location.

In case (b), both the quadratic and the bounding polynomial $\breve{f}_{p}(x)$ are initially of upward slope and downward curvature. Both functions will continue to increase until they reach their respective slope inflection points. Hence $\breve{f}_{p}(x)$ has no real root until after its inflection. Between its inflection point and the positive-real root of the downwardly curved quadratic, $\breve{f}_{p}(x)$ has one real root.

In case e) both functions are initially of positive slope and upward curvature. This condition is taken to be the absence of a root and the search stops. If continuing the search, the bounding polynomial $\breve{f}_{p}(x)$ will continue to increase until it passes first its curvature inflection followed by its slope inflection. After that, $\breve{f}_{p}(x)$ is monotone down if $\breve{f}_{3: p}(x)$ has at least one nonzero coefficient. It follows that $\breve{f}_{p}(x)$ has only one real root on the positive real line. If $\breve{f}_{p}(x)$ has not crossed below zero before the end of the allowed step length, the search is continued with the next step.

As the quadratic has no real root on that interval, $\breve{f}_{p}(x)$ would need to be sampled at increasing intervals to determine where it changes sign, marking the upper limit on a single real root. In case d), the quadratic is downward sloping but upward curving with no real roots. The bounding polynomial $\breve{f}_{p}(x)$ cannot be guaranteed to be monotone down, and Fig. 10 shows an example $\breve{f}_{p}(x)$ that has an inflection between negative and positive slope before it will eventually have another inflection making it monotone down. There is no simple means to judge the bound function to have a single real root on an interval. On this one case, and in exchange for reducing the order of the error term, the quadratic is maintained up to its minimum in Fig. 10, after which it is replaced by a constant of that minimum value. A nonzero $\breve{f}_{3: p}(x)$ makes the resulting piecewise function of the " $2-1$ bound" monotone down. It also gives the bound function negative slope at the location of the parabolic minimum. A single outward Newton-Raphson iteration using that slope finds the upper limit of an interval containing one real root. A zero $\breve{f}_{3: p}(x)$ results in a bound function that does not trend downward, and this condition is taken to be the absence of a root where the search stops. If continuing the search, the path is advanced to the allowed length of the current step, and the search is continued with the next step.

The final length of a step using series reversion is known before conducting the expansion, allowing the expansion to correct the error from the previous step. Such a corrector is not as easily combined with the predictor step here in the non-reversion method. Function $f(x)$ needs to be expanded at the start of the step to obtain its bounding polynomial $\breve{f}_{p}(x)$ giving a step limit not overrunning the zero. Reducing the step length to meet this limit under-applies any correction combined into the expansion of $f(x)$. The non-reversion method is therefore conducted with a separate, second-order corrector, the calculation time penalty being under $20 \%$ [27]. The algorithm below estimates the first zero of $\theta_{z}^{\{d\}}$, where $d$ is the order of the derivative of the angle of a passive joint.

method findZeroAngleDerivative(int $d$,

return boolean success)

\{

Apply corrector;

Conduct series expansion for predictor;

Evaluate $\theta_{z 1}^{\{d\}}$ from series coefficients;

$\theta_{z 0}^{\{d\}} \leftarrow \theta_{z 1}^{\{d\}} ; t_{0} \leftarrow$ tZeroBound $\left(\theta_{z}^{\{d\}}[0 \ldots p-d]\right)$;

while $\left(t_{0}>10^{-12} \& \& \theta_{z 1}^{\{d\}} \theta_{z 0}^{\{d\}}>\left|\theta_{z 0}^{\{d\}}\right| 10^{-10}\right)\{$

$t_{x} \leftarrow \max \operatorname{Step}()$;

// Limits steps approaching zero to $.14 t_{x}$

if $\left(t_{0} \geq .14 t_{x}\right)$

$\left\{t_{0} \leftarrow(1-.07) t_{x} ;\right.$ if $\left.\left(t_{0}>t_{x}\right) t_{0} \leftarrow t_{x} ;\right\}$

Update kinematic variables at $t_{0}$

from series expansion;

Apply corrector;

Conduct series expansion for predictor;

Evaluate $\theta_{z 1}^{\{d\}}$ from series coefficients;

\}

$t_{0} \leftarrow \operatorname{tZeroBound}\left(\theta_{z}^{\{d\}}[0 \ldots p-d]\right)$;

success $=t_{0}>0 \| \theta_{z 1}^{\{d\}} \theta_{z 0}^{\{d\}} \leq\left|\theta_{z 0}^{\{d\}}\right| 10^{-10} ;$

\}

Cite this article: P. Milenkovic (2022). "Optimal path crossing the orientation exclusion zone of a robot with offset wrist", Robotica 40, 191-212. https://doi.org/10.1017/S0263574721000461 